\title{
Wortgeografischer Wandel im Schweizerdeutschen. Sommersprossen, Küchenzwiebel und Schmetterling 70 Jahre nach dem SDS*
}

\author{
Britta Juska-Bacher (Basel)
}

\begin{abstract}
Since the beginning of the publication of the linguistic atlas of German-speaking Switzerland (Sprachatlas der deutschen Schweiz, SDS) in the early 1960s individual linguists collected contemporary material for comparison to investigate language change. However, due to time and money restrictions these studies were limited to small parts of the language area only. So far a description of tendencies concerning the entire Swiss German language area is missing.

Based on an online-survey of 5600 informants this investigation is the first to present word geographic data covering (almost) the whole German-speaking Switzerland. Comparing GISmaps of SDS and online data of the dialectal lexemes for freckles, onion and butterfly, language change over the last century becomes apparent, with striking convergence tendencies towards standard German, but also a Swiss German dialect expanding its range. Most of the dialect words mentioned in the SDS were preserved; some new were found. Thus, diversity of lexicon and creative language use are not endangered. Statistical analysis showed that younger speakers are more likely to deviate from the SDS. Less strong, but still significant were the influence of the parent's dialect and the duration of living in the dialect area, whereas gender had no influence.
\end{abstract}

\section{$1 \quad$ Einleitung}

Mit dem Sprachatlas der deutschen Schweiz (SDS; Erhebungen in den Jahren 1939-58) liegt für den Deutschschweizer Sprachraum umfangreiches Kartenmaterial zur Laut-, Wort- und Formengeografie der ersten Hälfte des 20. Jahrhunderts vor (zur Datierung cf. Hotzenköcherle 1962: 6). Auf der Grundlage dieser Kartensammlung wurden bereits in einer Reihe von Untersuchungen Vergleichsdaten - zu meist lautlichen Phänomenen - erhoben, um Sprachwandel im Schweizerdeutschen nachzuweisen (cf. z. B. Wolfensberger 1967; Bigler 1979; Christen 1988, 1998a; Schifferle 1995; Siebenhaar 2000, 2003, 2008). Da die Erfassung phonetischer Feinheiten linguistisch geschulter Interviewer bedarf, blieb man methodisch der direkten Erhebung des SDS verpflichtet und beschränkte sich, u. a. wegen begrenzter finanzieller wie zeitlicher Ressourcen, auf einen Ausschnitt des Sprachraums. Im Gegensatz dazu war die im Folgenden beschriebene Untersuchung darauf angelegt, wie der SDS den gesamten Deutschschweizer Raum zu erfassen. Um Dialektsprechende mit möglichst breiter regionaler Streuung bei überschaubarem zeitlichen Aufwand für die Teilnahme gewinnen zu können, ${ }^{1}$ wurde statt mit der direkten Befragung in Interviews mit der indirekten Erhebung in Form einer Online-Befragung gearbeitet. Daraus resultierend fiel die vermittelnde und Antworten fixierende Instanz des linguistisch geschulten Interviewers und damit die Möglichkeit der zuverlässigen Erhebung phonetischer Feinheiten weg. Stattdessen wurde Material aus demjenigen linguistischen Bereich erhoben, der den Befragten am besten bewusst und von ihnen am leichtesten festzuhalten ist, nämlich dem Wortschatz (cf. auch Elspass/Möller 2006: 149; Eichhoff 1982: 550).

\footnotetext{
* Die Autorin dankt den beiden anonymen Gutachtern für ihre wohlwollende Kritik und die konstruktiven Verbesserungsvorschläge zu einer früheren Version dieses Beitrags.

1 Für den SDS wurde mit einem dichten Ortsnetz von 573 Punkten gearbeitet (Hotzenköcherle 1962: 92).
} 
Ziel der Untersuchung war es, exemplarisch aktuelle wortgeografische Daten zu erheben, die über den Vergleich mit SDS-Karten Aussagen zu Veränderungstendenzen in der Deutschschweizer Dialektlandschaft der letzten gut 70 Jahre erlauben. ${ }^{2}$

\section{Material und Methoden}

Als Material für die Befragung wurden 25 SDS-Wortkarten aus den Bänden IV bis VI ausgewählt. Bedingung für die Auswahl war erstens, dass es sich beim Bezeichneten um heute noch allgemein bekannte Gegenstände, Tätigkeiten, Berufe, Tiere etc. handelte (d. h. die grosse Gruppe des landwirtschaftlichen Vokabulars wurde nicht berücksichtigt). Da es nicht darum ging, ausgehend von diesen Karten Verallgemeinerungen auf die übrigen Wortkarten oder gar alle SDS-Karten vorzunehmen, wurde nicht versucht, eine für die eine oder andere Gruppe repräsentative Auswahl zu treffen. Stattdessen wurden SDS-Karten mit im Hinblick auf Veränderungen "interessanten" Verteilungen ausgewählt (z. B. Karten mit einer klaren Zweiteilung oder mit besonders klaren Verteilungen von drei oder mehr Dialektwörtern oder Karten mit auffällig zahlreichen unterschiedlichen regionalen Bezeichnungen). Es soll daher an dieser Stelle noch einmal betont werden, dass es sich bei den im Folgenden beschriebenen Ergebnissen (hier vorgestellt die Ergebnisse zu Sommersprossen, Küchenzwiebel und Schmetterling) lediglich um Einzelbeispiele handelt, anhand derer einige Veränderungstendenzen im Schweizer Dialektwortschatz aufgezeigt werden können.

Wie im SDS wurde auch in der Online-Befragung nach der onomasiologischen Methode gearbeitet. Die Probanden wurden anhand von Umschreibung, Abbildung, Lückentext oder standardsprachlicher Vorgabe nach dem in ihrem Dialekt gebräuchlichsten Wort gefragt. Dabei wurde halboffen, d. h. mit einer Reihe von Vorgaben (je nach Zahl der Nennungen im SDS zwei bis sieben Begriffe, nämlich die bei der SDS-Befragung am häufigsten genannten; lautliche Unterschiede wurden dabei nicht berücksichtigt) und einer offenen Kategorie für mögliche weitere Nennungen, gearbeitet. ${ }^{3}$ Im Einleitungstext des Fragebogens wurden die Probanden explizit gebeten, das in ihrer Mundart am häufigsten verwendete Wort zu markieren. Zudem sollten sie die betreffende Mundart, für welche die Antworten galten (inklusive Postleitzahl), sowie verschiedene soziodemografische Daten angeben. Der Fragebogen ${ }^{4}$ wurde auf dem Server des Deutschen Seminars der Universität Zürich abgelegt und über verschiedene Printmedien, eine Radiosendung sowie die Online-Ausgabe einer Gratiszeitung wurde zum Ausfüllen aufgerufen..$^{5}$ An dieser Befragung, die von November 2007 bis April 2008 lief, beteiligten sich rund 5600 Probanden von 1258 verschiedenen Ortspunkten (in dieser Untersuchung wiedergegeben als Postleitzahlbezirke) der Deutschschweiz. ${ }^{6}$ Die Verteilung der Probanden auf die Postleitzahlbezirke ist in Abb. 1 dargestellt.

2 Da das Interesse Heinrich Baumgartners und Rudolf Hotzenköcherles archaisierend war (Hotzenköcherle 1962: 5), wurden gezielt ältere, ortsansässige Probanden ausgewählt (meist zwischen 51 und 80 Jahre alt, cf. Hotzenköcherle 1962: 121), die explizit nach einem alten Sprachstand gefragt wurden (Trüb 1982: 153). Eine Datierung der Probandendaten ist, zumal die Erhebungen in einem Zeitraum von 20 Jahren erfolgten, nur tendenziell möglich. Hotzenköcherle (1962: 6) geht nach grober Schätzung von den ersten Jahrzehnten des 20. Jahrhunderts aus.

3 So lautete beispielsweise Frage 21 nach der Bezeichnung für den Schmetterling im jeweiligen Dialekt:

"Aus der Raupe entwickelt sich ein ...

$\square$ Schmätterling

$\square$ Summervogel

$\square$ Müllervogel

$\square$ Fifalter

$\square$ Anders, nämlich:

4 Mein Dank für Anmerkungen zum Fragebogen gilt Dr. Barb Breustedt, Prof. Dr. Elvira Glaser sowie den Teilnehmenden des von mir im Herbstsemester 2007 an der Universität Basel (Deutsches Seminar) durchgeführten Kurses Einführung in die Methoden der empirischen Sprachwissenschaft.

5 Ich danke Balz Spörri (Sonntagszeitung vom 11.11.2007), Christian Schmid (Schnabelweid, Aufrufe in den Sendungen vom 22.11. und 29.11.2007 sowie Interview am 27.12.2007) und Daniel Huber (Print-Ausgabe von 20 Minuten vom 10.01.2008 und Online-Ausgaben vom 07. bis 11.01.2008) für ihr Interesse und die Verbreitung des Aufrufs im jeweiligen Medium.

6 Ihnen allen sei für die Teilnahme ganz herzlich gedankt. 


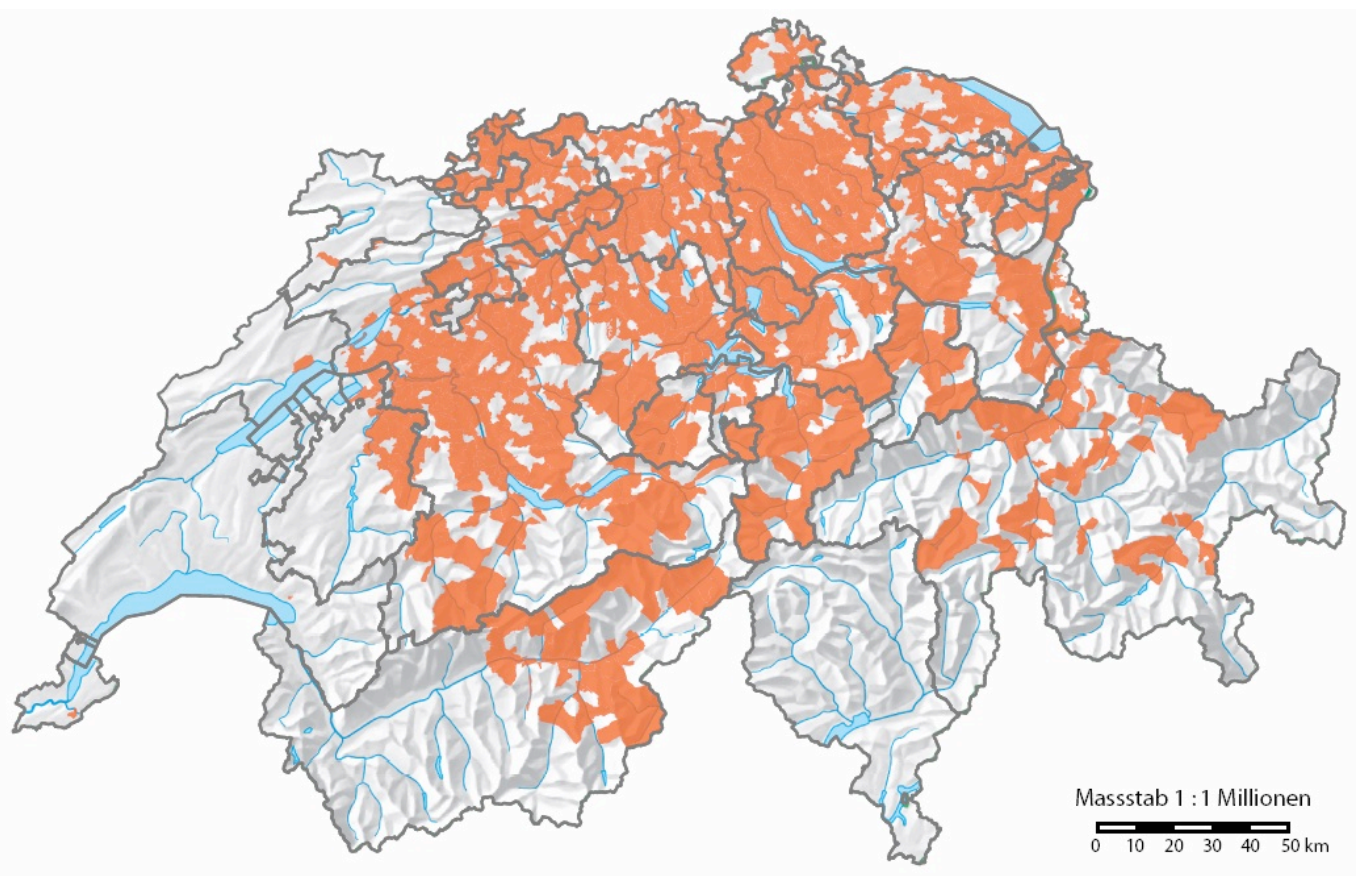

Abb. 1: Überblick der in der Online-Befragung abgedeckten Postleitzahlbezirke

Die eingegangenen Daten wurden einerseits deskriptiv ausgewertet, d. h. die Angaben der Probanden wurden Postleitzahlbezirken zugeordnet und kartografisch dargestellt (s. Kapitel 3), um sie mit SDS-Karten vergleichen und wortgeografischen Wandel feststellen zu können. Andererseits wurden die Angaben mit Hilfe von Verfahren der schliessenden Statistik (verallgemeinertes gemischtes lineares Modell = VGLM) ausgewertet, um herauszufinden, ob bestimmte soziodemografische Faktoren einen Einfluss auf die Tendenz zu modernerem Wortgebrauch der Probanden haben (s. Kapitel 4).

Es seien an dieser Stelle zusammenfassend einige grundsätzliche methodische Unterschiede zwischen der direkten Erhebung des SDS und der indirekten Erhebung des Online-Fragebogens und ihre Folgen angesprochen, die bei der Interpretation der Daten im Sinne einer Längsschnittstudie zu berücksichtigen sind (ausführlicher dazu cf. Juska-Bacher 2009).

Der Wechsel zur indirekten Methode führte zum Wegfall der Vermittlungsinstanz (s. Einleitung) und legte damit eine Beschränkung auf wortgeografisches Material und zudem einen vergleichsweise bescheidenen Umfang nahe. Verallgemeinerungen der Online-Ergebnisse und Rückschlüsse auf andere linguistische Bereiche (z. B. lautliche Veränderungen) sind - auch aufgrund der nicht repräsentativen Zusammenstellung - nicht möglich, waren auch nicht intendiert. Diese Veränderung gegenüber dem SDS beschränkte lediglich das zur Auswahl stehende Untersuchungsmaterial.

Die Auswahl der Probanden erfolgte nicht wie beim SDS gerichtet (nämlich nach den ältesten und ortsansässigen Dialektsprechern), sondern ungerichtet, indem online eine möglichst grosse Zahl potentieller Probanden zur Teilnahme aufgerufen wurde. Während die SDSProbanden deutlich fortgeschrittenen Alters waren (meist zwischen 51 und 80 Jahre, cf. Hotzenköcherle 1962: 121), gaben zur Online-Befragung - wie dies auch in anderen Untersuchungen festgestellt wurde (cf. Elspaß/Möller 2006: 146; Dräger/Juska-Bacher 2009: 169, Fussnote 4) - überwiegend jüngere Probanden ihre Stimme ab (mehr als $50 \%$ waren jünger als 30 Jahre alt, s. Abb. 2). 


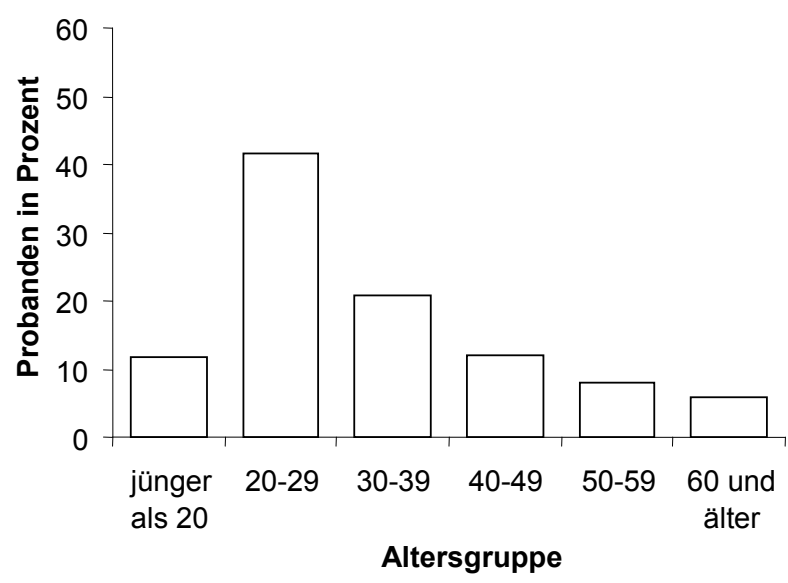

Abb. 2: Altersverteilung der Probanden der Online-Befragung zum schweizerdeutschen Wortschatz

Diese Altersverschiebung der Probandengruppe beeinflusst die Bestimmung des zeitlichen Abstands zwischen beiden Untersuchungen. Einerseits wird davon ausgegangen, dass die SDS-Daten grob die Verhältnisse in den ersten Jahrzehnten des 20. Jahrhunderts repräsentieren (cf. Hotzenköcherle 1962: 6). Andererseits ist aufgrund des hohen Anteils junger Probanden in der Online-Befragung - unter der Annahme ihres fortschrittlicheren Wortgebrauchs (s. dazu die Ergebnisse in Kapitel 4.1) - davon auszugehen, dass der Dialektstand in der Online-Befragung tendenziell ein wenig fortschrittlicher wiedergegeben wird, als er tatsächlich ist. Damit liegen die Sprachdaten der beiden Untersuchungen zeitlich deutlich weiter voneinander entfernt als ihre Erhebungsdaten. ${ }^{7}$

An der Online-Befragung beteiligten sich rund 5600 Probanden (d. h. gut 0,1\% der Deutschschweizer Bevölkerung). Damit äusserten sich im Vergleich zur SDS-Befragung fast zehnmal so viele Probanden zu den einzelnen Fragen. Im Vergleich zum SDS konnten die Probanden mehr als doppelt so vielen Ortspunkten bzw. Postleitzahlenbezirken zugeordnet werden (nämlich 1258 statt 573), wobei immerhin etwa 85\% der SDS-Ortspunkte abgedeckt wurden. Dieser Deckungsgrad weist darauf hin, dass nicht jeder einzelne Ortspunkt 1:1 verglichen, grobe Tendenzen aber problemlos bestimmt werden können. Aufgrund der grossen Teilnehmendenzahlen äussern sich in der Online-Befragung im Gegensatz zum SDS häufig mehrere Probanden eines Ortspunktes, so dass eine Bestimmung der ortsüblichen Form weniger subjektiv als im SDS (mit nur einem Probanden pro Ortspunkt und Frage) sein dürfte.

Während die SDS-Exploratoren offen, d. h. ohne Vorgabe einer Antwortauswahl arbeiteten, wurde die Online-Befragung wegen der leichteren Erfass- und Auswertbarkeit der grossen Datenmengen halboffen durchgeführt. Es ist möglich, dass die Vorgaben zu Echoformen geführt haben. In diesem Fall würden die Abweichungen vom SDS in den erhobenen Daten tendenziell geringer ausfallen (da die SDS-Wörter im Fragebogen der Online-Erhebung vorgegeben waren) als in der Sprachwirklichkeit bzw. die nachweisbaren Wandeltendenzen wären geringer, als sie es tatsächlich sind.

Unter Berücksichtigung dieser methodischen Unterschiede und ihrer Auswirkungen bietet die Online-Befragung eine vielversprechende und realisierbare Möglichkeit, Wortschatzwandel im gesamten Deutschschweizer Raum empirisch zu belegen. Die im folgenden Kapitel beschriebenen - neben den Veränderungstendenzen deutlich erkennbaren - Übereinstimmungen wortgeografischer Grobverteilungen beider Erhebungen sprechen für die Reliabilität dieser Methodenkombination (s. die Vergleiche der Gesamtkarten in den Abbildungen 3 und 4, 8 und 9 sowie 12 und 13 sowie die noch grösseren Übereinstimmungen zwischen SDS und den Einzelkarten in Kapitel 3).

\section{Wortgeografische Verteilungen in SDS- und Online-Erhebung und sich daraus}

\footnotetext{
7 Zum Erreichen möglichst grosser Parallelität von Online- und SDS-Erhebung wäre es eine Möglichkeit gewesen, auch die Online-Probanden nach einem möglichst alten Dialektstand zu befragen. Da eine sinnvolle Auswertbarkeit aufgrund der Altersheterogenität der Probanden (im Gegensatz zu den SDS-Probanden) nicht möglich schien, wurde darauf verzichtet.
} 


\section{ergebende Veränderungstendenzen}

Bevor in den Unterkapiteln Kartenmaterial zu Sommersprossen (3.1), Küchenzwiebel (3.2) und Schmetterling (3.3) gezeigt wird, sei im Vorab etwas zur Entstehung und Beschaffenheit der Karten gesagt. ${ }^{8}$ Aus den Online-Befragungsdaten wurden auf der Basis der Postleitzahlbezirke (auf Gemeindebasis) farbige Flächenkarten erstellt. Dafür wurden den einzelnen dialektalen Bezeichnungen Farben zugeordnet und ihrer Nennung die jeweiligen Postleitzahlbezirke in dieser Farbe eingefärbt. Aus Gründen der Vergleichbarkeit wurden auch die betreffenden SDS-Punktsymbolkarten in entsprechende farbige Flächenkarten umgewandelt.

Beim Vergleich der Karten sind zwei Punkte zu beachten. Erstens lagen von den OnlineProbanden Daten von doppelt so vielen Ortspunkten vor (s. Kapitel 2), so dass sich auf ihrer Grundlage deutlich vollere Kartenbilder ergeben. Zweitens ist zu berücksichtigen, dass bei der Online-Befragung im Gegensatz zum SDS vielfach mehrere Probanden pro Ortspunkt Angaben gemacht haben, so dass teilweise eine Auswahl aus diesen Mehrfachnennungen notwendig wurde.

Es wurden drei verschiedene Kartentypen erstellt:

1. Den SDS-Karten entsprechende Gesamtkarten (s. Abb. 4, 9 und 13), in denen die von den Probanden genannten Dialektwörter zur Bezeichnung von Sommersprossen, Küchenzwiebel bzw. Schmetterling enthalten sind. Dabei wurde nur die jeweils häufigste Nennung eines Postleitzahlbezirks verzeichnet (Auswahl).

2. Einzelkarten zu den genannten Dialektwörtern (Abb. 5-7, 10 und 11 und 14-16), in denen alle Nennungen der Probanden berücksichtigt wurden. D. h. ein Postleitzahlbezirk kann in den verschiedenen Einzelkarten zu einer Wortfrage unterschiedlich eingefärbt sein (keine Auswahl) ${ }^{9}$

3. Veränderungskarten zu den einzelnen Wörtern (s. Anhang 1-3), in denen mit jeweils drei Farben gearbeitet wurde, um Übereinstimmungen und Unterschiede zwischen SDS- und Online-Befragung zu kennzeichnen. Auch in diesen Karten wurden alle Nennungen berücksichtigt (keine Auswahl).

\subsection{Sommersprossen (SDS-Karte IV 43)}

Die SDS-Karte (umgewandelt in eine Flächenkarte s. Abb. $3^{10}$ ) zeigt hinsichtlich der dialektalen Bezeichnungen der standardsprachlichen Sommersprossen einen deutlichen OstWest-Gegensatz, wie er für eine Reihe von SDS-Karten sowohl für lautliche, morphologische wie lexikalische Phänomene festgestellt wurde. Im Westen (bis zur Ostgrenze des Kantons Aargau, westlicher Teil des Kantons Zug) ist relativ einheitlich Laubfläcke belegt. Der östliche Landesteil zeigt sich uneinheitlicher: Hier besteht einerseits ein Nord-Süd-Gegensatz, ${ }^{11}$ wobei im Süden ebenfalls Laubfläcke genannt wurde. Im Norden wurden für den an den westlichen Laubfläcke-Bereich angrenzenden Teil (Kantone Zürich, südliches Sankt Gallen, Zug, Schwyz) Merzefläcke angegeben, im Nordosten hingegen eine Reihe weiterer Komposita mit Merze- als erstem und -dräck, -sprig(g)ele, -tupfe etc. als zweitem Glied. Summersprosse wurde lediglich viermal verstreut über das Deutschschweizer Gebiet genannt (in der Karte mit türkisen Kreisen markiert).

${ }^{8}$ Für ihre Erstellung gebührt mein aufrichtiger Dank Friedjoff Trautheim und Prof. Dr. Robert Weibel (Geografisches Institut der Universität Zürich).

${ }^{9}$ In Abbildung 10 bspw. sind in der Online-Daten-Karte (rechts) verschiedene Postleitzahlbezirke im Kanton Zürich braun eingefärbt, weil die Probanden Zibele angaben, in Abbildung 11 (rechts) ist, besonders in der östlichen Hälfte, eine Reihe derselben Postleitzahlbezirke blau eingefärbt, weil Probanden zu diesen Orten auch Böle als dialektale Entsprechung für Küchenzwiebel nannten.

${ }^{10}$ Der Übersichtlichkeit halber ist hier eine Auswahl erfolgt, indem nur die am häufigsten genannten Dialektbegriffe in die Karte übernommen wurden. Im SDS weiterhin verzeichnet sind: Merzepletter, -flätter, -blueme, -glöggli, -rüseli, -böle, -brägel und -sprosse.

${ }^{11}$ Der bevorzugte Verlauf von Isoglossen in Nord-Süd- wie auch in West-Ost-Richtung wurde für das Schweizerdeutsche wiederholt hervorgehoben (cf. z. B. Hotzenköcherle 1986 und Haas 2000). 


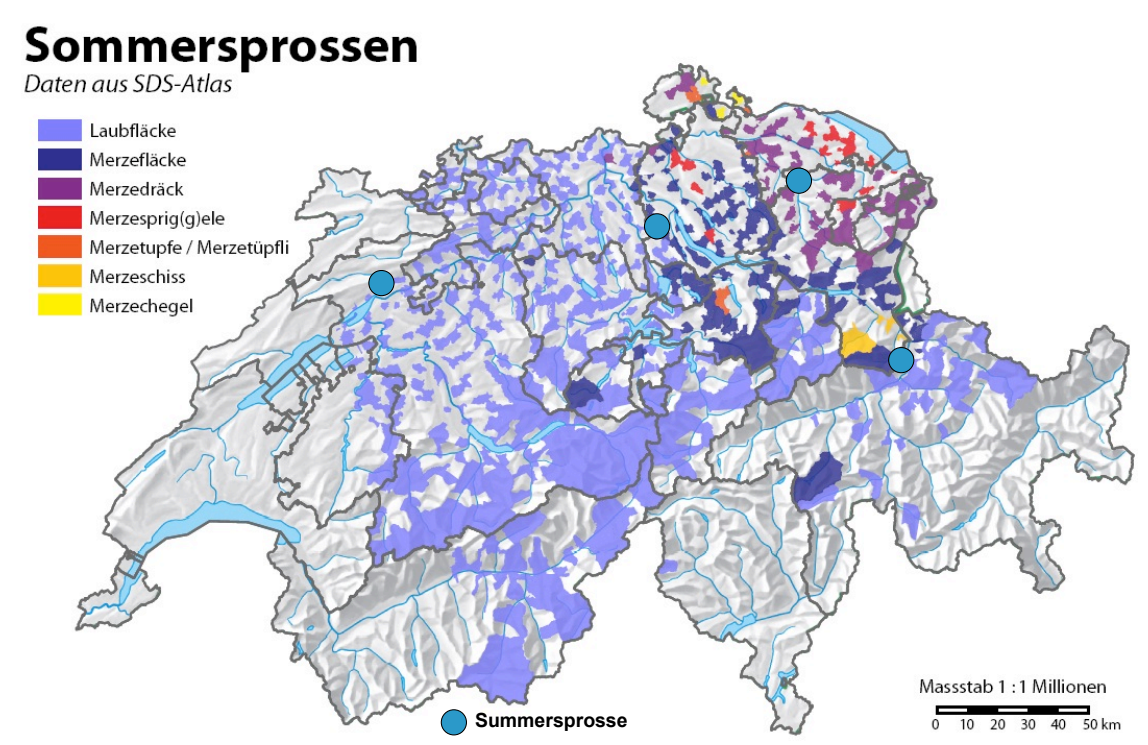

Abb. 3: In eine Flächenkarte umgewandelte SDS-Karte (IV 43) für die dialektale Bezeichnung von Sommersprossen

Betrachtet man die kartografische Darstellung der rund 70 Jahre später ermittelten OnlineDaten (Abb. $4^{12}$ ), so ist die Grobgliederung der SDS-Karte noch deutlich erkennbar. Auch in dieser Befragung wurden Laubfläcke für die westliche Hälfte des Sprachraums angegeben, allerdings scheint sich die Grenze ein wenig nach Westen verschoben zu haben. Im Osten herrschen Komposita mit Merze- vor. Merzefläcke wurden besonders in den Kantonen Zürich, Zug, Schwyz, Glarus sowie in Graubünden und Sankt Gallen genannt. Im Nordosten sind wie auf der SDS-Karte die Komposita Merzedräck, -sprig(g)ele, -tupfe belegt. Ein grosser Teil des Sprachraums ist neben dieser Gliederung mit einem Netz von Summersprosse-Nennungen überzogen.

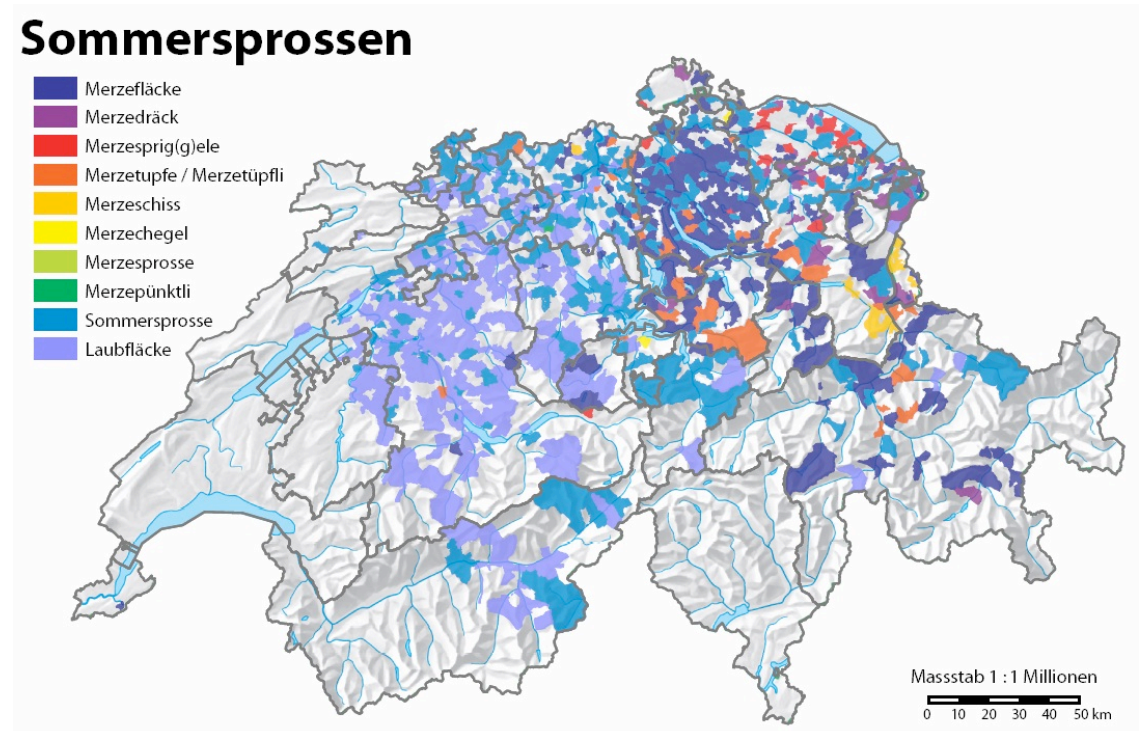

Abb. 4: Karte zur aktuellen dialektalen Bezeichnung von Sommersprossen (Grundlage: Online-Befragung)

Um die Veränderungen einerseits noch deutlicher $\mathrm{zu}$ visualisieren und andererseits in der Gesamtkarte durch die Auswahl verdeckte Zustände offenzulegen, werden im Folgenden die SDS- (Erhebungszeitpunkt $t_{1}$ ) und die Online-Befragungsdaten (Erhebungszeitpunkt $t_{2}$ ) pro

\footnotetext{
${ }^{12}$ Der Übersichtlichkeit halber wurden für die Erstellung der Karten, die auf der Online-Befragung basierten, nur Bezeichnungen verwendet, die von mindestens 5 Probanden genannt wurden. Für die Sommersprossen ausserdem genannt wurden u. a. Brägeli, Bschütitupf, Dräcksprüzer, Flü̈̈geschiss, Güllesprüz, Läberäfleck, Merzeblueme, -stärne, Sunnespriggel.
} 
Lexem in Einzelkarten visualisiert (hier nur für die drei am weitesten verbreiteten Wörter). Dafür wurden nicht nur die häufigsten, sondern alle Nennungen der Probanden berücksichtigt, d. h. es wurden alle Postleitzahlbereiche eingefärbt, in denen Laubfläcke im Fragebogen angegeben wurde. Abb. 5 stellt die Verbreitung dieses Wortes zu beiden Erhebungszeitpunkten (eigentlich -zeiträumen) dar. Im Vergleich dieser Kartenbilder ist zu erkennen, dass Laubfläcke eine sehr ähnliche Ausdehnung zeigt. Abgesehen von vereinzelten Nennungen im Kanton Zürich ${ }^{13}$ stimmt die Ostgrenze in der nördlichen Hälfte des Sprachraums fast überein. Aus der südöstlichen Hälfte hat sich dieses Wort tendenziell zurückgezogen (besonders in den Kantonen Graubünden, Glarus, Uri).

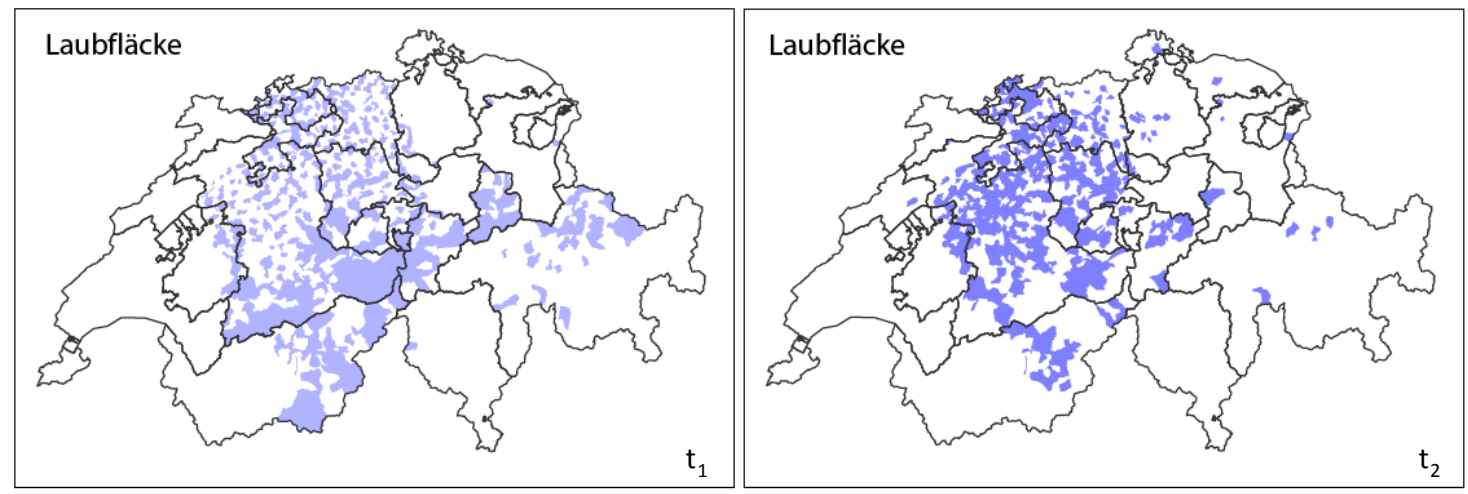

Abb. 5: Die Verbreitung von Laubfläcke im SDS (links) und auf der Grundlage der Online-Daten (rechts)

Abb. 6 verdeutlicht die Ausbreitung der dialektalen Merzefläcke von der östlich an die Laubfläcke-Region angrenzenden Region (mit Schwerpunkt in den Kantonen Zürich, Zug, Schwyz) sowohl nach Norden (Schaffhausen), Westen (bis Basel, Bern, Fribourg), Süden (Uri) als auch nach Osten (Graubünden, Sankt Gallen und Thurgau). Abgesehen vom Wallis wurde dieses Dialektwort in allen Kantonen mehrfach belegt.

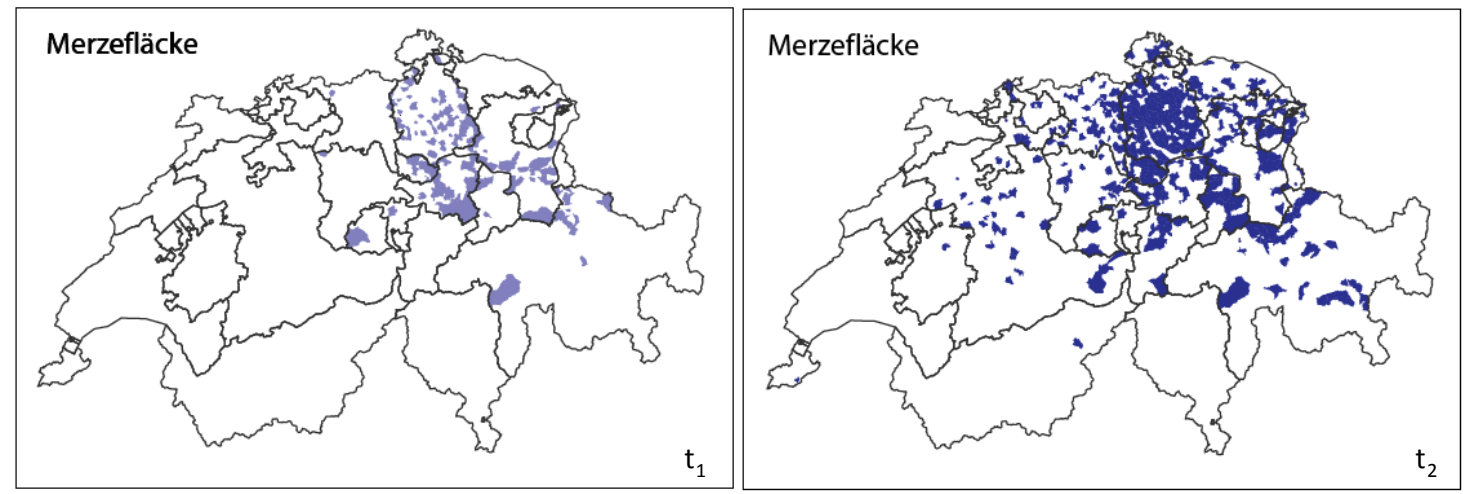

Abb. 6: Die Verbreitung von Merzefläcke im SDS (links) und auf der Grundlage der Online-Daten (rechts)

Abb. 7 belegt die auffälligste wortgeografische Veränderung zwischen den beiden Erhebungen, nämlich die von Norden her bis ins Wallis, nach Uri und Graubünden erfolgte "Invasion" der standardsprachlichen Sommerspossen (lautlich an die dialektalen Verhältnisse angepasst von den Probanden als Summersprosse wiedergegeben). In allen deutschsprachigen Kantonen wurde diese Bezeichnung als am häufigsten verwendete gewählt. Am Anfang dieses Kapitels wurde darauf hingewiesen, dass für die Erstellung der Einzelkarten alle Nennungen der Probanden berücksichtigt wurden. Dazu zählen auch Mehrfachangaben der Probanden, die laut Eingangstext des Fragebogens nur gestattet waren, wenn diese gleich häufig verwendet wurden. In diesem Zusammenhang ist anzumerken, dass weniger als $3 \%$

\footnotetext{
13 Je grösser die Teilnehmerzahlen aus einem Ort oder Kanton, desto grösser die Wahrscheinlichkeit, dass ein Proband einen für die betreffende Region abweichenden Ausdruck angibt. Da aus dem Kanton Zürich mehr als 1800 Probanden stammten, erstaunt es nicht, dass einzelne von ihnen auch die hier eher unüblichen Laubfläcke genannt haben.
} 
der Probanden, die Summersprosse genannt haben, daneben ein weiteres Wort angaben, d. h. $97 \%$ haben sich eindeutig für Summersprosse als gebräuchlichste Bezeichnung entschieden. Dieses Lexem scheint also nicht als Variante neben den in der Standardsprache nicht verwendeten Wörtern (Laubfläcke, Märzefläcke etc.) zu stehen, sondern sich bei einem relativ grossen Teil der Schweizerdeutschsprechenden als geläufigste Bezeichnung für die Pigmentflecken durchgesetzt zu haben. Im Online-Fragebogen war dieses Lexem wegen seines seltenen Vorkommens im SDS (s. Abb. 3) nicht vorgegeben und musste von den Probanden aktiv als von ihnen in ihrem Dialekt am häufigsten verwendetes Wort unter "Anders, nämlich" eingefügt werden. Unter der Annahme von Echoformen könnte das tatsächliche Vorkommen dieses Lexems noch höher liegen als auf der Karte angezeigt (s. Kapitel 2).

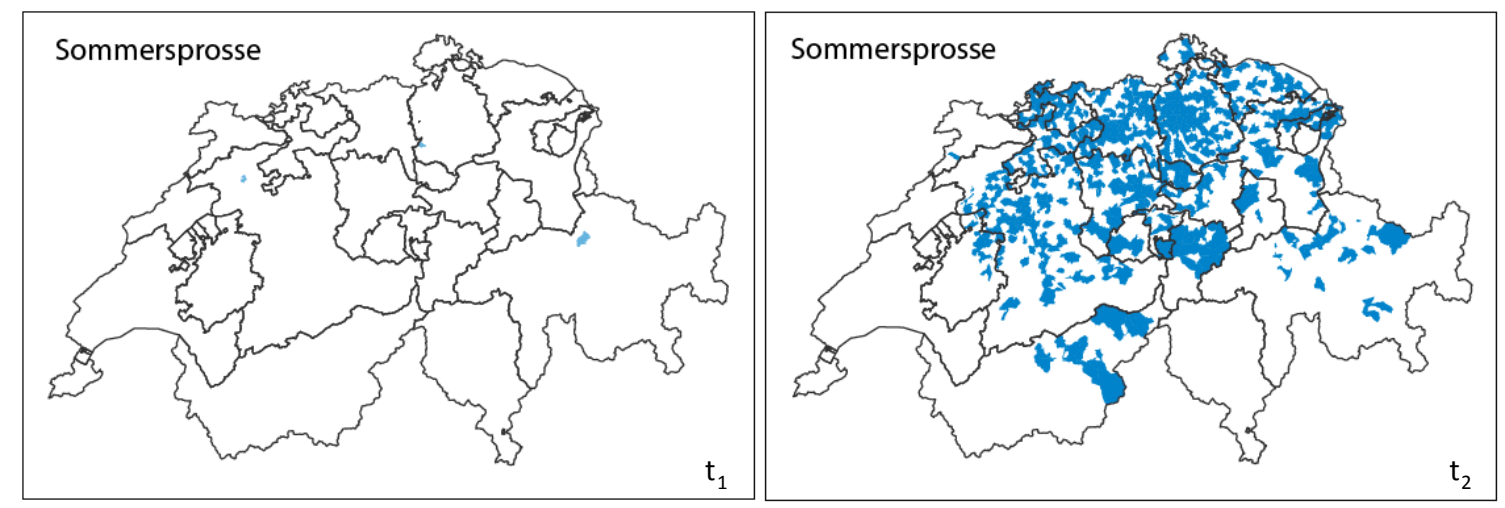

Abb. 7: Die Verbreitung von Summersprosse im SDS (links) und auf der Grundlage der Online-Daten (rechts)

Da die unterschiedliche Zahl und teilweise Lage der Ortspunkte in beiden Untersuchungen sowie das mögliche Auftreten von Echoformen bei seltener verwendeten Wörtern schwerer ins Gewicht fällt und ggf. die Ergebnisse beeinflussen kann, wird an dieser Stelle auf eine detaillierte Beschreibung ihrer Entwicklung und damit verbunden eine Aussage über die dialektale Vielfalt verzichtet. Stattdessen sind im Anhang Veränderungskarten beigefügt, in denen die Daten beider Erhebungen für die einzelnen Dialektwörter in Beziehung gesetzt werden. Aus ihnen ist ersichtlich, dass Formen wie Merzedräck, -sprig(g)ele und -tupfe durchaus noch lebendig sind und gegenwärtig sogar ein grösseres Verbreitungsgebiet zu haben scheinen als im SDS.

Zusammenfassend lässt sich auf der Grundlage der aus den Online-Daten entstandenen Karte (s. Abb. 4) für die wortgeografischen Verteilungen der dialektalen Bezeichnungen von Sommersprossen herausstellen:

1. Die Grobstrukturen der SDS-Karte (s. Abb. 3) sind deutlich wiederzuerkennen. Dies betrifft vor allem die West-Ost-Gliederung (Laubfläcke, Merzefläcke weitere Komposita mit Merze-), weniger deutlich die Nord-Süd-Gliederung im östlichen Landesteil (Laubfläcke auf dem Rückzug). Während die Einzelkarte (Abb. 5, s. auch Anhang 1) zeigt, dass Laubfläcke wie im SDS bis zur östlichen Kantonsgrenze des Aargaus sowie im westlichen Teil des Kantons Zug angegeben wurde, ist diese Grenze in der Gesamtkarte (Abb. 4) nach Westen verschoben. Dies weist darauf hin, dass Laubfläcke am östlichen Rand zwar durchaus noch verwendet wird, offensichtlich aber von Merzefläcke (s. Abb. 6) und Summersprosse (s. Abb. 7) zurückgedrängt wird.

2. Auffälligste Veränderung ist, dass sich über den grössten Teil des Deutschweizer Raums ein Netz von Sommersprossen-Nennungen gelegt hat. Damit hat die standardsprachliche Bezeichnung als äusserst erfolgreiches "invasives" Lexem gegenüber dem SDS massiv an Terrain gewonnen - ohne aber das Kartenbild vollständig zu beherrschen.

3. Neben dem standardsprachlichen Einfluss lässt sich mit dem Ausstrahlen von Merzefläcke vom Kerngebiet Zürich - Zug - Schwyz in alle Himmelsrichtungen aber eben- 
falls sehr deutlich eine Verschiebung innerhalb des im SDS verzeichneten dialektalen Gefüges feststellen.

4. Die Ausbreitung von Summersprosse und Merzefläcke hat die übrigen hier berücksichtigten Begriffe (weitere Merze-Komposita) mit vergleichsweise geringerer und gestreuterer Verbreitung nicht verdrängen können (s. Abb. 4 sowie Anhang 1). Im Vergleich mit der SDS-Karte wurde als neue dialektale Bezeichnung Merzepünktli genannt.

5. Mit der Verwendung von Summersprosse als überregionale Form in weiten Teilen des Sprachraums ist das Gesamtkartenbild weniger klar gegliedert als im SDS. Dies kann auch mit der unterschiedlichen Anlage der beiden Studien in Zusammenhang stehen: die Online-Befragung sollte die aktuell verwendeten Formen erfassen, während mit der SDS-Erhebung ein möglichst alter Dialektstand festgehalten werden sollte, standardnahe Formen wurden daher unter Umständen bei der Erhebung als weniger relevant eingeschätzt und gar nicht erfasst.

\subsection{Küchenzwiebel (SDS-Karte VI 179)}

Die SDS-Karte (als Flächenkarte s. Abb. 8) zeigt für die dialektalen Bezeichnungen der Küchenzwiebel eine klare West-Ost-Zweiteilung des Sprachraums. Im Westen (bis zum Osten der Kantone Aargau und Luzern, bis zur östlichen Kantonsgrenze Berns) wurde Zibele/ Zübele, im Osten (ab östliches Aargau und Luzern sowie ab Unterwalden und Uri) Böle/Bölle angegeben.

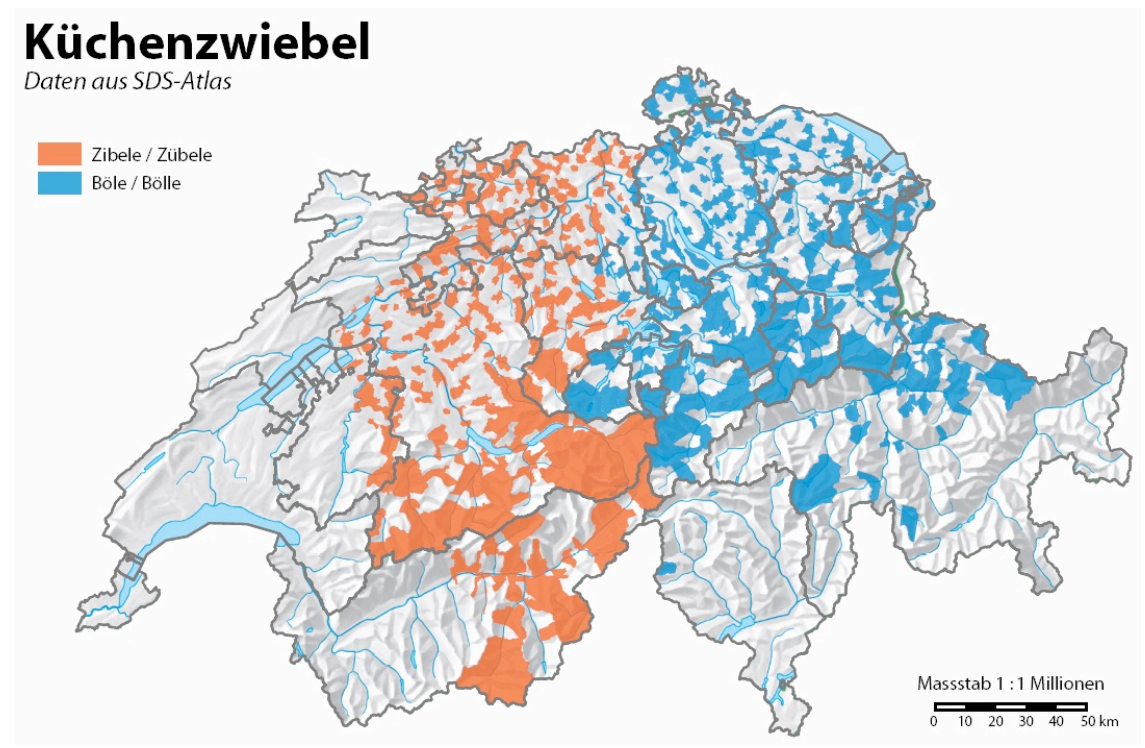

Abb. 8: In eine Flächenkarte umgewandelte SDS-Karte (VI 179) für die dialektale Bezeichnung von Küchenzwiebel

Die kartografische Darstellung der Online-Daten (s. Abb. 9) zeigt eine ähnliche Grobgliederung mit Zibele im Westen und Böle im Osten. Allerdings gibt es keine klare Grenze zwischen den Gebieten mehr, sondern Zibele wurde im östlichen Landesteil besonders auch im Norden (Kantone Zürich, Thurgau und nördliches Sankt Gallen) und in Graubünden als häufigste verwendete Bezeichnung genannt. Hingegen kam Böle in der westlichen Region nur sehr vereinzelt vor. 


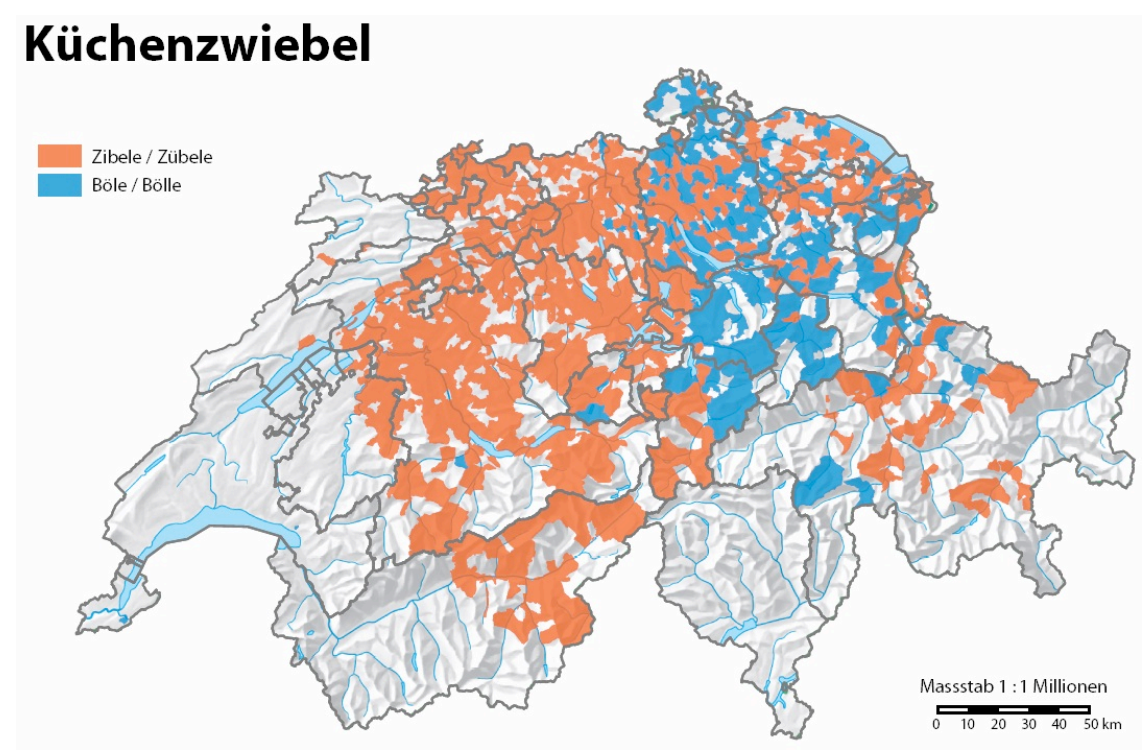

Abb. 9: Karte zur aktuellen dialektalen Bezeichnung von Küchenzwiebel (Grundlage: Online-Befragung)

Die Einzelkarten (cf. Abb. 10), in denen wiederum alle (nicht nur die häufigsten) Nennungen berücksichtigt wurden, stellen die Verbreitung von Zibele in beiden Erhebungen gegenüber. Die Ausdehnung nach Osten ist besonders dicht im Norden, zieht sich aber durch die gesamte östliche Landeshälfte.
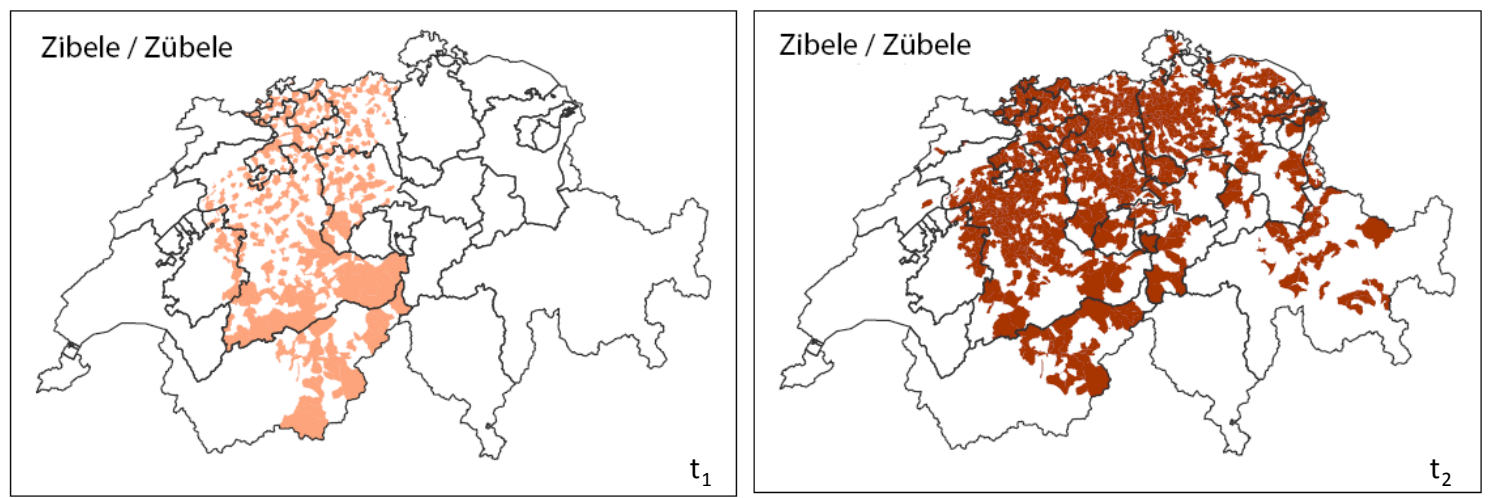

Abb. 10: Die Verbreitung von Zibele im SDS (links) und auf der Grundlage der Online-Daten (rechts)

Der direkte Vergleich der Böle-Einzelkarten zeigt die Beschränkung von Böle auf die östliche Landeshälfte. Böle tritt im Zibele-Gebiet nur sehr vereinzelt auf, so dass die Westgrenze (abgesehen von einzelnen Nennungen im Kanton Bern) sehr ähnlich geblieben ist.
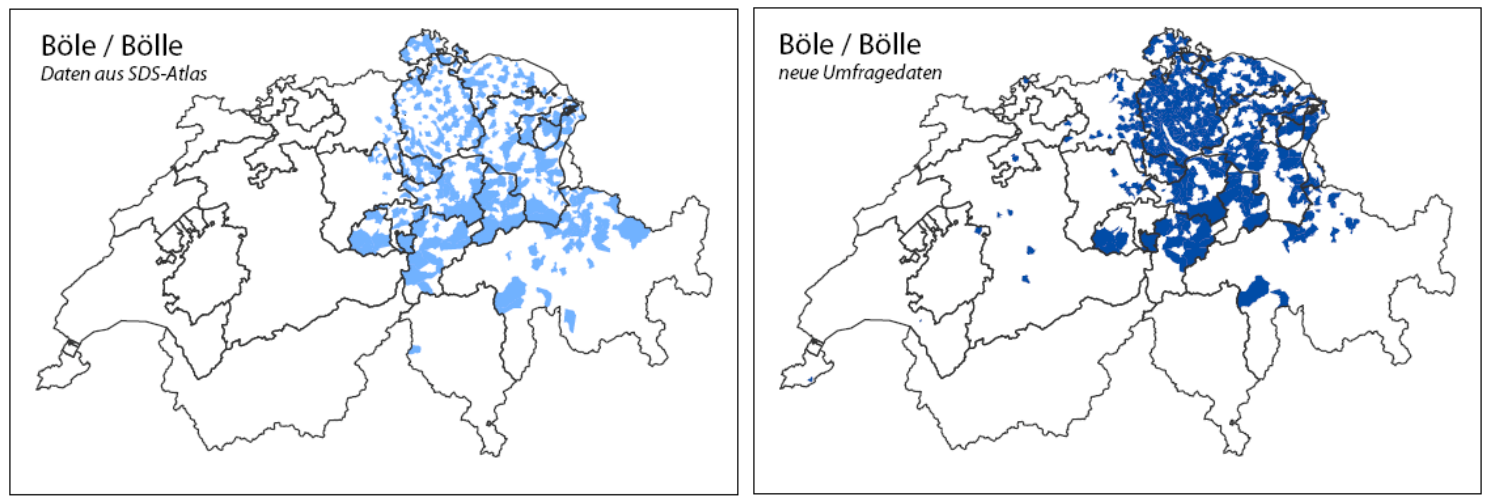

Abb. 11: Die Verbreitung von Böle im SDS (links) und auf der Grundlage der Online-Daten (rechts) 
Die Ergebnisse zur Küchenzwiebel lassen sich wie folgt zusammenfassen:

1. Die Grobstruktur der SDS-Karte (s. Abb. 8) mit einem West-Ost-Gegensatz der zwei Wörter ist deutlich wiederzufinden. Allerdings lässt sich keine klare Grenze zwischen der Verwendung der Begriffe mehr ziehen.

2. Dies ist darauf zurückzuführen, dass die Zibele-Verwendung (cf. das standardsprachliche Lexem Zwiebel) sich sehr deutlich auf Kosten von Böle nach Osten ausgebreitet und damit überregionale Verwendung gefunden hat, während Böle sich nicht nach Westen ausgebreitet hat.

3. Vergleicht man die Westgrenze der Böle-Nennungen auf der Einzelkarte (Abb. 11, s. auch Anhang 2) mit ihrer Grenze auf der Gesamtkarte (Abb. 9), so fällt auf, dass auf der Einzelkarte der Grenzverlauf demjenigen der SDS-Karte entspricht. Im Bereich der Kantonsgrenzen Aargau - Zürich, in Zug, an den Grenzen Luzern - Schwyz, Nidwalden - Uri wird Böle zwar noch genannt, aber Zibele ist der Gesamtkarte zufolge inzwischen deutlich die häufigere Form geworden. Dieser Kartenvergleich macht die Tendenz des Zurückdrängens von Böle in Richtung Osten sehr deutlich.

\subsection{Schmetterling (SDS-Karte VI 237)}

Aus der SDS-Karte (als Flächenkarte s. Abb. 1214) ist hinsichtlich der dialektalen Entsprechungen für den standardsprachlichen Schmetterling eine Nord-Süd-Gliederung ersichtlich. In den nördlichen Kantonen (bis zum nördlichsten Bern, Luzern, Obwalden, Nidwalden, nördliches Schwyz, nördliches Sankt Gallen) wurde Summervogel angegeben, im Süden meist Fifalter (bzw. diverse lautliche Varianten), daneben in den Kantonen Bern, im südlichen Nidwalden, Glarus, in Sankt Gallen, Appenzell und Graubünden auch Schmätterling.

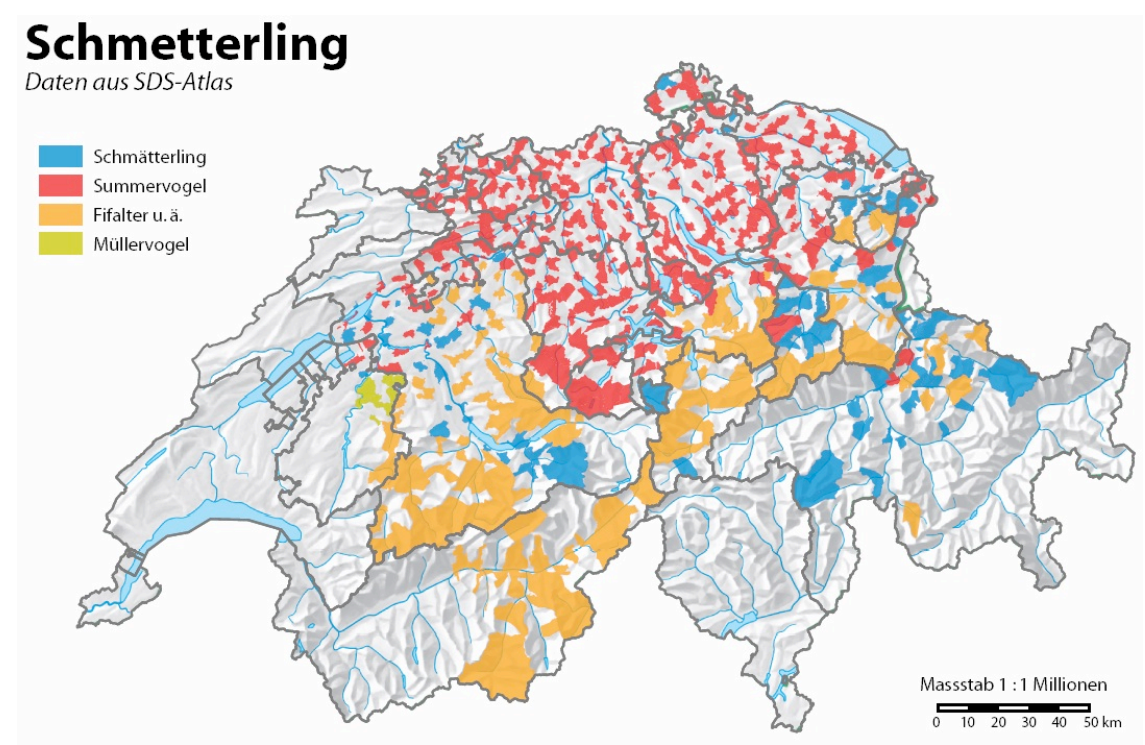

Abb. 12: In eine Flächenkarte umgewandelte SDS-Karte (VI 237) für die dialektale Bezeichnung von Schmetterling

Die auf der Grundlage der Online-Daten erstellte Karte (s. Abb. 1315) zeigt ein deutlich verändertes Bild. Dominiert wird das aktuelle Gesamtbild von Schmätterling-Nennungen, die auf der SDS-Karte fast ausschliesslich verstreut im Süden des Sprachraums vorkamen (in den nördlichen Kantonen nur sehr vereinzelte Nennungen). Sie werden in der nördlichen Hälfte des Sprachraums (besonders in den Kantonen Aargau, Zürich, Luzern, Obwalden) immer wieder von Summervogel, in der südlichen (in den Kantonen Fribourg, Bern, Wallis, Schwyz,

\footnotetext{
14 Im SDS ausserdem angeführt wurden vereinzelte Nennungen von: Sunnevogel, Müller(vogel) und Toggeli.

15 In der Online-Befragung weniger als fünfmal genannt wurden bspw.: Schmättervogu, Härgottsfautär, Fligholzer, Müllervogu. Mehr als fünfmal, aber nie als häufigste Bezeichnung (und daher in der Gesamtkarte nicht enthalten) genannt wurde im Vergleich zum SDS neu Schmätzgi.
} 
Uri, Appenzell und Graubünden) von Fifalter unterbrochen (insofern ist die SDS-Verteilung noch deutlich erkennbar). Der im SDS noch für das nordöstliche Fribourg verzeichnete Müllervogel wurde in der Online-Befragung nirgendwo (im Kanton Fribourg) als ortspunkthäufigste Form genannt und ist deshalb in der Gesamtkarte nicht enthalten (s. dazu allerdings die Darstellung in Anhang 3).

\section{Schmetterling}

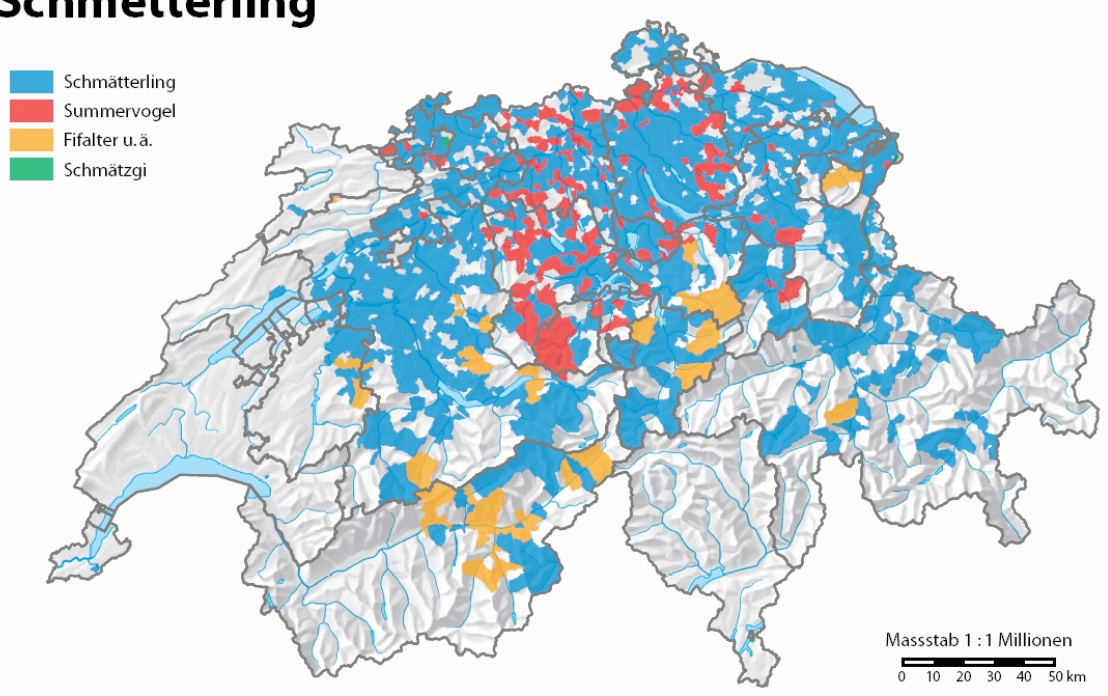

Abb. 13: Karte zur aktuellen dialektalen Bezeichnung von Schmetterling (Grundlage: Online-Befragung)

Der Vergleich der Summervogel-Einzelkarten (Abb. 14) zeigt, dass dieses Dialektwort von den Online-Probanden etwa für das gleiche Gebiet wie von den SDS-Probanden angegeben wurde (s. auch Anhang 3). Aus der Gesamtkarte ist allerdings ersichtlich, dass sie vielerorts nicht mehr als häufigste angegeben wurde (stattdessen Schmätterling).

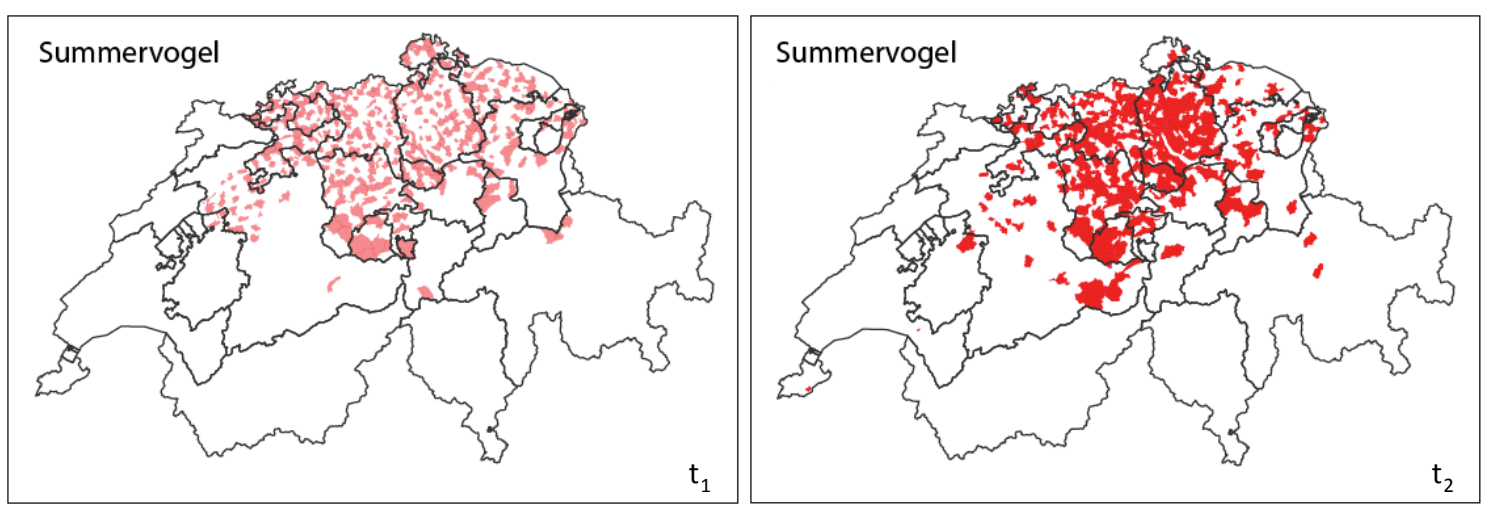

Abb. 14: Die Verbreitung von Summervogel im SDS (links) und auf der Grundlage der Online-Daten (rechts)

Hingegen geht aus dem Vergleich der Fifalter-Karten hervor, dass diese Bezeichnung in der Online-Befragung (besonders in den Kantonen Bern, Wallis, Glarus, Sankt Gallen und Graubünden) tendenziell seltener angegeben wurde als im SDS. 

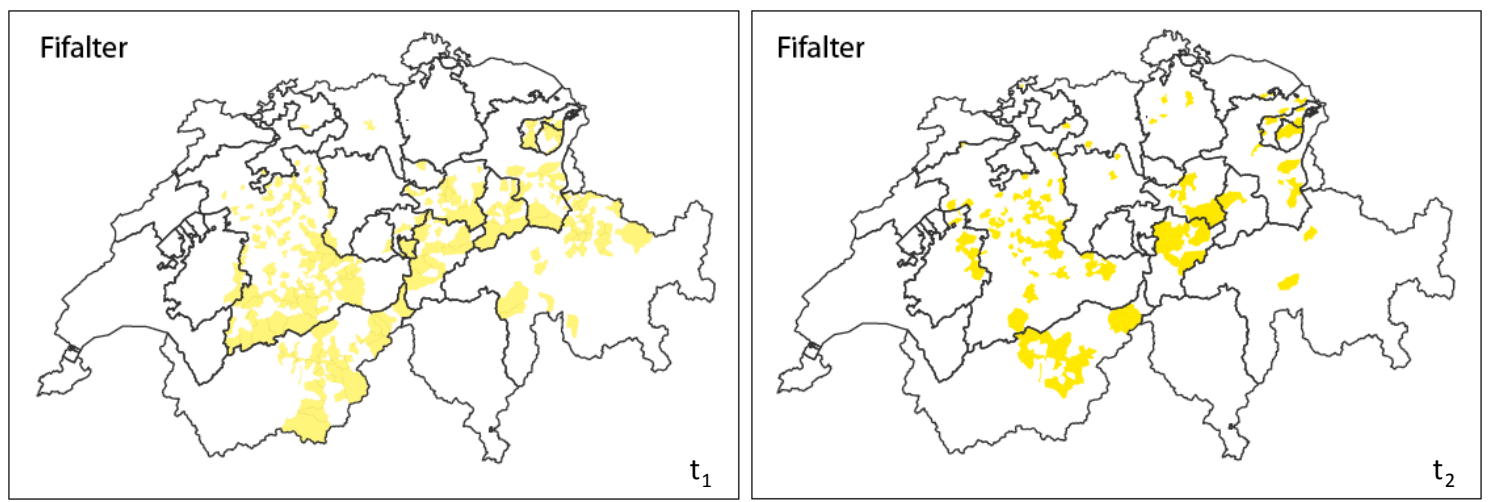

Abb. 15: Die Verbreitung von Fifalter im SDS (links) und auf der Grundlage der Online-Daten (rechts)

Während Schmätterling im SDS noch auf Einzelnennungen, grösstenteils im Süden des Sprachraums beschränkt war, zeigt die Einzelkarte zu den Online-Daten, dass diese Bezeichnung praktisch für fast jeden Ortspunkt bezeugt wurde (Abb. 16, deutlich im Vergleich mit Abb. 1, in der die Abdeckung der Postleitzahlbezirke gezeigt ist).
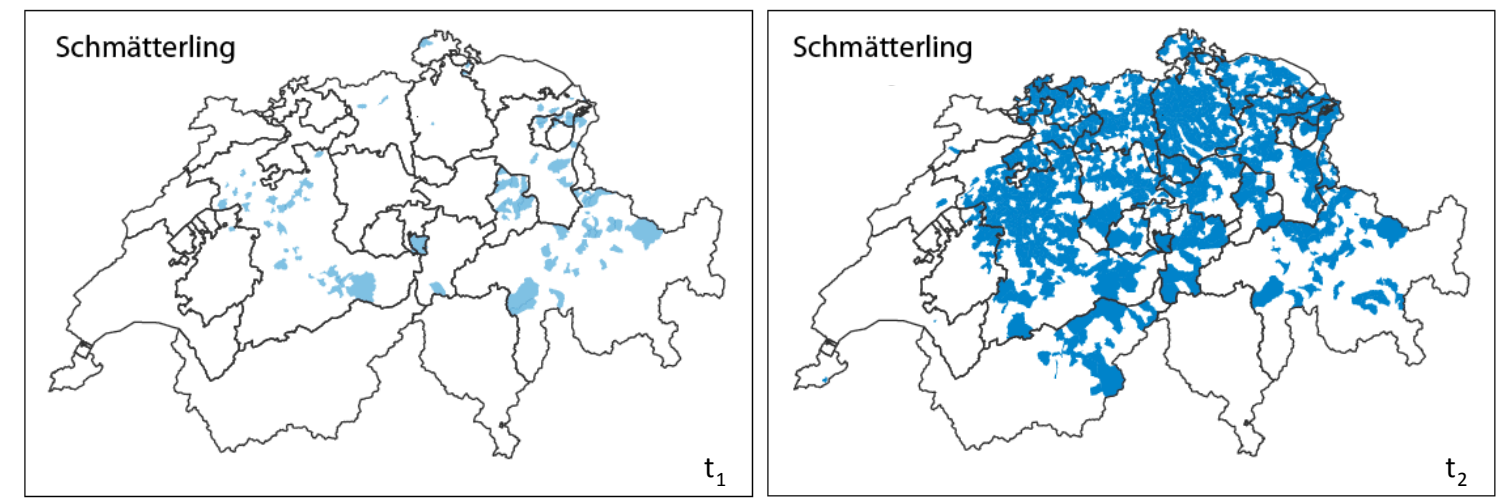

Abb. 16: Die Verbreitung von Schmätterling im SDS (links) und auf der Grundlage der Online-Daten (rechts)

Die auf der Grundlage der Online-Erhebung erstellten Karten zu den wortgeografischen Verteilungen der Schweizer dialektalen Bezeichnungen des Schmetterlings lieferten zusammengefasst folgende Ergebnisse:

1. In den Einzelkarten (Abb. 14-16), in denen alle genannten Dialektbegriffe berücksichtigt wurden, lässt sich die Grobstruktur der SDS-Karte (Summervogel im Norden, Fifalter mit eingestreutem Schmätterling im Süden) zwar noch erkennen, in der Gesamtkarte (Abb. 13) aber ist sie durch ein dichtes Netz von SchmätterlingNennungen überdeckt. ${ }^{16}$

2. Dieses auch in der Standardsprache verwendete Wort wurde im Gegensatz zum SDS, wo es nur eingestreut ins Fifalter-Gebiet vorkam, an (fast) jedem Ortspunkt, in vielen als gängigste Bezeichnung genannt.

3. Wie aus den Einzelkarten (Abb. 14-16) hervorgeht, hat die massive Verbreitung des Schmätterlings als gegenwärtig am häufigsten verwendete Bezeichnung die übrigen (Summervogel, Fifalter und Müllervogel) bisher nicht vollständig verdrängt.

4. Neben den hier erwähnten dialektalen Bezeichnungen wurde im Vergleich zum SDS in den Kantonen Basel, Zürich und Bern etwa ein Dutzend Mal (unabhängig vom Alter der Probanden) Schmätzgi angeführt (da nirgendwo als häufigste, erscheint sie nicht in der Gesamtkarte).

16 Auf einen Ersatz des Summervogels durch den Schmätterling weist bereits Christen (1998b: 60) hin, ohne allerdings das Ausmass dieser Verdrängung und die Betroffenheit der übrigen Wörter angegeben zu können. 
5. Mit der weiten, das heisst überregionalen Verbreitung des Schmätterlings ist auch dieses Gesamtkartenbild im Vergleich zum SDS in sehr viel weniger klare, deutlich von einander abzugrenzende Regionen gegliedert.

Nachdem in diesem Kapitel die Karten der zwei Erhebungszeiträume gegenübergestellt und für Sommersprossen, Küchenzwiebel und Schmetterling zahlreiche Veränderungen festgestellt wurden, soll im folgenden Kapitel statistisch getestet werden, ob sich Zusammenhänge zwischen soziodemografischen Daten und dem Abweichen der Probanden vom SDS feststellen lassen.

\section{Welche Faktoren beeinflussen die Tendenz zu einem modernen Wortgebrauch der Probanden?}

Für die statistische Auswertung wurden die Online-Probanden aus Gründen der direkten Vergleichbarkeit auf diejenigen reduziert, die Angaben zu SDS-Ortspunkten gemacht hatten. Damit blieben rund 3000 Probanden für die Tests übrig. Aufgrund dieser relativ grossen Probandenzahl sollten generelle Muster, so vorhanden, deutlich nachweisbar sein. Als abhängige Variable galt das Kriterium "Abweichung der dialektalen Bezeichnungen für Sommersprossen, Küchenzwiebel und Schmetterling gegenüber dem SDS" (ja vs. nein). Als unabhängige oder erklärende Variable wurden folgende Faktoren berücksichtigt:

1. Alter (intervallskaliert)

2. Ortsansässigkeit (hier definiert als mindestens 10 Jahre im Geltungsbereich der Ortsmundart wohnhaft, in zwei nominalskalierten Stufen): ja - nein

3. Dialekt der Mutter (entspricht der vom Probanden angegebenen Ortsmundart, in zwei nominalskalierten Stufen): ja - nein

4. Dialekt des Vater (entspricht der vom Probanden angegebenen Ortsmundart, in zwei nominalskalierten Stufen): ja - nein

5. Geschlecht (zwei nominalskalierte Stufen): weiblich - männlich

Auf der Grundlage der Daten zu den Dialektwörtern für Sommersprossen, Küchenzwiebel und Schmetterling wurden verallgemeinerte gemischte lineare Modelle (VGLM) durchgeführt. Wichtig ist, dass die Zahl der erforderlichen Probanden umso grösser sein muss, je komplexer das Versuchsdesign ist. Auf der anderen Seite gilt: je grösser die Probandenzahl, desto leichter wird die Signifikanzgrenze erreicht. Es ist daher bei der Arbeit mit vielen Probanden sehr wichtig, nicht nur den p-Wert, sondern die tatsächlichen Unterschiede zwischen den Gruppen, d. h. die tatsächlichen Effekte zu berücksichtigen. Im Unterschied zu allgemeinen linearen Modellen (ALM), die nur mit festen Variablen - von denen ein Zusammenhang mit Antwortvariablen vermutet wird - arbeiten, werden bei den gemischten Modellen neben den festen auch sogenannte zufällige Faktoren (random effects) berücksichtigt. Zufälliger Faktor in diesem Modell war der Postleitzahlbezirk (Berücksichtigung der ersten zwei Stellen), zu welchem eine Ortsmundart gehört. Probanden aus demselben Postleitzahlbezirk machen in der Regel Angaben zum gleichen Dialekt und können deshalb nicht als unabhängig gelten (möglicherweise verhalten sich Probanden aus einem Postleitzahlbezirk im Wallis grundsätzlich anders als Probanden aus Schaffhausen).

Die VGLM ergaben die in den Tabellen 1-3 wiedergegebenen Signifikanzen:

\begin{tabular}{lcc}
\hline Faktor & Effekt & Signifikanz \\
\hline Alter & -0.05 & $<0.001$ \\
Ortsansässigkeit & -0.27 & 0.004 \\
Dialekt der Mutter & -0.28 & 0.008 \\
Dialekt des Vaters & -0.29 & 0.008 \\
Geschlecht & 0.005 & 0.950 \\
\hline
\end{tabular}

Tabelle 1: VGLM zum Einfluss soziodemografischer Daten auf die Tendenz der Online-Probanden vom SDS abzuweichen. Material: Sommersprossen-Daten. Anzahl der Gewährsleute: $\mathbf{n}=3318$.

Faktor $\quad$ Effekt Signifikanz




\begin{tabular}{lrr}
\hline Alter & -0.05 & $<0.001$ \\
Ortsansässigkeit & -0.18 & 0.120 \\
Dialekt der Mutter & -0.35 & 0.003 \\
Dialekt des Vaters & -0.46 & $<0.001$ \\
Geschlecht & 0.19 & 0.054 \\
\hline
\end{tabular}

Tabelle 2: VGLM zum Einfluss soziodemografischer Daten auf die Tendenz der Online-Probanden vom SDS abzuweichen. Material: Küchenzwiebel-Daten. Anzahl der Gewährsleute: $\mathbf{n}=3318$.

\begin{tabular}{lcc}
\hline Faktor & Effekt & Signifikanz \\
\hline Alter & -0.04 & $<0.001$ \\
Ortsansässigkeit & 0.17 & 0.122 \\
Dialekt der Mutter & -0.35 & 0.004 \\
Dialekt des Vaters & -0.07 & 0.577 \\
Geschlecht & -0.09 & 0.325 \\
\hline
\end{tabular}

Tabelle 3: VGLM zum Einfluss soziodemografischer Daten auf die Tendenz der Online-Probanden vom SDS abzuweichen. Material: Schmetterling-Daten. Anzahl der Gewährsleute: $\mathbf{n}=3318$.

\subsection{Einfluss des Alters der Probanden}

Ausgangspunkt dieser statistischen Untersuchung bildete Hypothese 1, derzufolge jüngere Sprecher(innen) sich als sprachlich flexibler erweisen sollten als ältere. Die Hypothese gründet sich einerseits darauf, dass älteren Probanden generell eine höhere Dialektkompetenz bescheinigt wird (cf. z. B. Niebaum/Macha 2006: 184) und andererseits in der Literatur verschiedentlich die Tendenz herausgestellt wurde, dass jüngere Probanden zu sprachgeografisch weiter verbreiteten Formen tendieren als ältere (cf. z. B. Wolfensberger 1967: 214; Christen 1988: 207; Hofer 1997: 220; Siebenhaar 2008: 191). Die VGLM ergaben, dass das Alter ${ }^{17}$ in allen drei Datensätzen einen hochsignifikanten Einfluss auf die Tendenz zu einem moderneren Wortgebrauch der Probanden hatte, und zwar dahingehend, dass Probanden mit zunehmendem Alter weniger Abweichungen gegenüber dem SDS aufwiesen $(p<0.001$, Effekt zwischen -0.04 bis -0.05, s. Tabellen 1-3). Die durchschnittliche Abweichung vom SDS in Abhängigkeit von der Altersgruppe (welche allerdings im Gegensatz zum VGLM den Zufallsfaktor Postleitzahlbezirk nicht berücksichtigt!) zeigt drei sehr ähnliche, fast lineare Kurven (Abb. 16), d. h. mit zunehmendem Alter werden proportional weniger Abweichungen vom SDS realisiert. Unter 20-Jährige wichen mit ihren Angaben zwischen rund 40 bis $60 \%$ vom SDS ab, während nur 10 bis gut $20 \%$ der Probanden im Alter von über 60 Jahren Abweichungen zeigten.

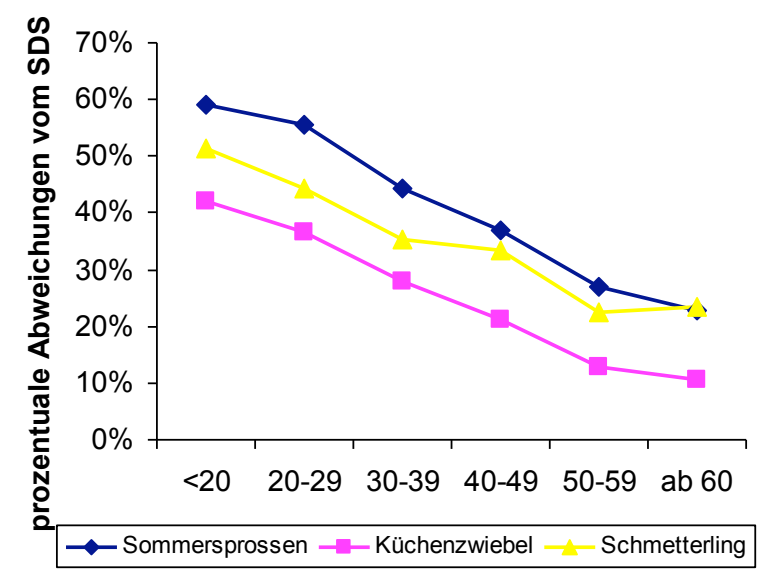

Abb. 17: Durchschnittliche Abweichung von SDS-Bezeichnungen für Sommersprossen in Prozent, in Abhängigkeit von der Altersgruppe

17 Zur Altersverteilung der Probanden in der Gesamtstichprobe s. Abbildung 1. 
Die Hypothese zur Abhängigkeit der Abweichung vom SDS vom Alter konnte damit sehr deutlich bestätigt werden.

\subsection{Einfluss der Ortsansässigkeit der Probanden}

Als Hypothese 2 wurde davon ausgegangen, dass ortsansässige Probanden weniger Abweichungen vom SDS zeigen als Probanden, die entweder nicht mehr am Ort wohnen, für den sie Auskunft gegeben haben, bzw. erst kürzere Zeit dort ortsansässig sind. Als "ortsansässig" galten hier Probanden, die seit mindestens 10 Jahren an dem Ort wohnen, über deren Mundart sie Auskunft gegeben haben. ${ }^{18}$ Gestützt wurde diese Hypothese durch Arbeiten wie die Wolfensbergers (1967: 214), der zeigte, dass Ortsansässige weniger überregionale Formen verwenden als Nicht-Ortsansässige.

Anders als beim Alter zeigten die VGLM hinsichtlich dieses Faktors unterschiedliche Ergebnisse. Das Signifikanzniveau wurde nur bei den Sommersprossen-Daten erreicht (s. Tabellen 1-3), d. h. die Nicht-Ortsansässigen wichen häufiger vom SDS ab als die Ortsansässigen. Dass der Effekt der Ortsansässigkeit nur für den Sommersprossen-Datensatz bestätigt werden konnte und damit in dieser Untersuchung deutlich geringer als erwartet war, so dass Hypothese 2 nur bedingt bestätigt werden kann, überrascht. Da aus der quantitativ soliden Grundlage eine hohe Aussagekraft der statistischen Tests (eine hohe "statistische Power") resultiert, ist davon auszugehen, dass - gäbe es auch für die übrigen Daten einen deutlichen Unterschied zwischen ortsansässigen und nicht-ortsansässigen Probanden - dieser hätte ausgewiesen werden sollen.

\subsection{Einfluss des Dialekts der Eltern}

Hypothese 3 ging davon aus, dass Probanden, die denselben Dialekt sprechen wie die Eltern, sprachlich eher konservativ sind und daher weniger vom SDS abweichen als die übrigen (zur Familie als Mundartgruppe cf. z. B. Wolfensberger 1967: 179-181). In den VGLM erwiesen sich der Dialekt der Mutter und des Vaters in den meisten Fällen tatsächlich als signifikant. ${ }^{19}$ Ein deutlicher Einfluss des mütterlichen Dialekts konnte in allen drei Datensätzen nachgewiesen werden (s. Tabellen 1-3), die Bedeutung des väterlichen Dialekts reichte von nichtsignifikant (Schmetterling: $\mathrm{p}=0.58$ ) über signifikant (Sommersprossen: $\mathrm{p}=0.008$ ) bis hochsignifikant (Küchenzwiebel: p < 0.001). Die Effekte waren immer negativ, d. h. Probanden, die den gleichen Dialekt sprechen wie ihre Eltern, weichen seltener von den SDS-Bezeichnungen $a b$.

Zur Veranschaulichung seien noch einmal die Mittelwerte der zwei Gruppen in einem Säulendiagramm dargestellt (auch hier ist der Zufallsfaktor Postleitzahlbezirk nicht berücksichtigt!; s. Abb. 18 und 19). Die Diagramme zeigen, dass Probanden mehr Abweichungen vom SDS aufweisen, wenn sie angaben, einen anderen Dialekt zu sprechen als die Mutter und der Vater.

\footnotetext{
18 Von den hier berücksichtigten Probanden lebten $68 \%$ seit 10 Jahren an dem Ort, über dessen Mundart sie Auskunft gegeben hatten.

19 Von den in der statistischen Auswertung einbezogenen Probanden gaben $72 \%$ an, den gleichen Dialekt zu sprechen wie die Mutter, und $75 \%$, den gleichen Dialekt wie der Vater.
} 


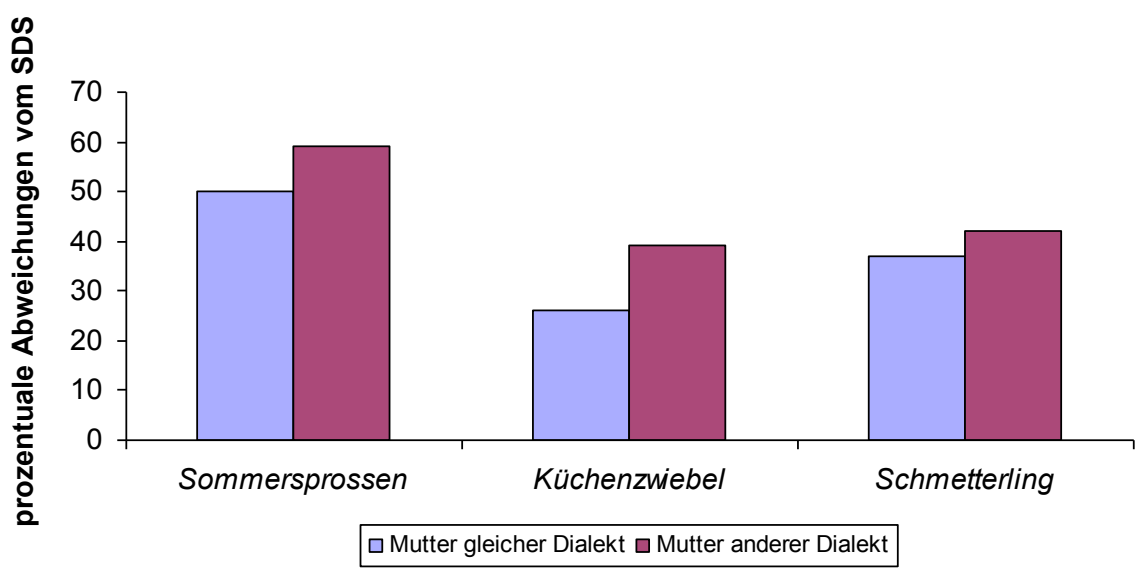

Abb. 18: Abweichung vom SDS in Prozent in Abhängigkeit von der Übereinstimmung des Dialekts der Probanden mit dem Dialekt ihrer Mütter

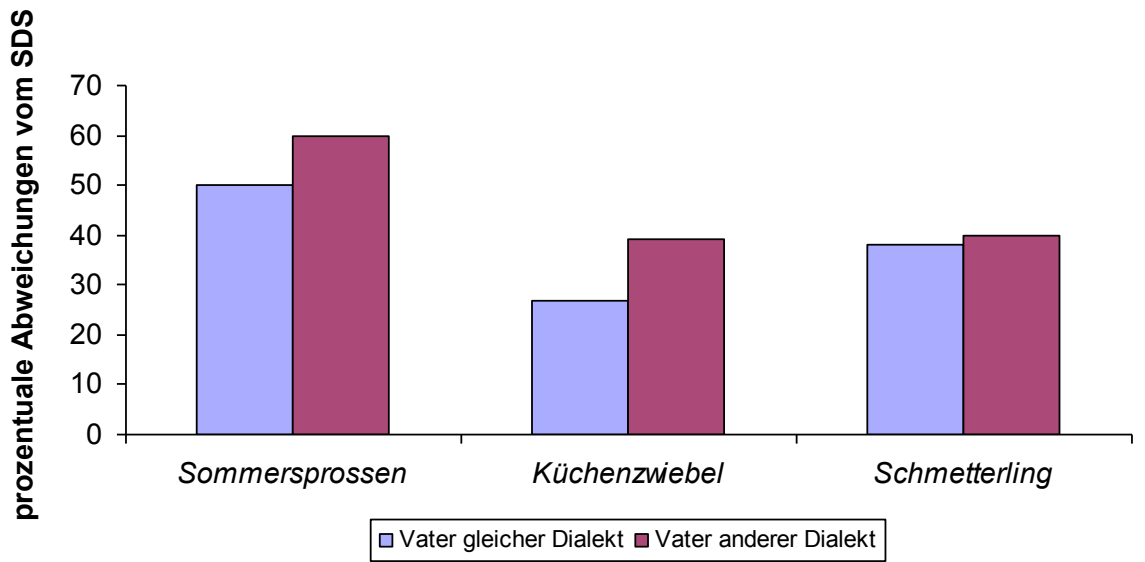

Abb. 19: Abweichung vom SDS in Prozent in Abhängigkeit von der Übereinstimmung des Dialekts der Probanden mit dem Dialekt ihrer Väter

Hypothese 3 konnte damit bestätigt werden.

\subsection{Einfluss des Geschlechts der Probanden}

Hinsichtlich des Geschlechts wurde in Hypothese 4, die sich u. a. auf Untersuchungen von Wolfensberger (1967: 186) und Hofer (1997: 207) stützt, davon ausgegangen, dass es keine nennenswerten Unterschiede zwischen Frauen und Männern gibt.

Dass der Einfluss des Geschlechts sich in keinem der VGLM als signifikant erwies (p-Werte zwischen 0.95 und 0.54 , s. Tabellen 1-3), bestätigte Hypothese 4.

\section{$5 \quad$ Diskussion}

\subsection{Wortgeografische Veränderungstendenzen}

Neben der deutlichen Wiedererkennbarkeit der Grobgliederungen der SDS-Karten (besonders der West-Ost- und der Nord-Süd-Gliederung) in den Karten der Online-Erhebung und der Übereinstimmung der Verbreitungsräume einiger Dialektwörter konnten verschiedene wortgeografische Veränderungstendenzen festgestellt werden. Dies erstaunt nicht, hatte doch bereits Wolfensberger (1967: 214) in den 1960er Jahren im Vergleich mit den SDS-Daten punktuell (für die Gemeinde Stäfa im Kanton Zürich) eine "ausgeprägte Umbruchs-Situation" des Schweizerdeutschen festgestellt, die sich in der "stellenweise unerhörten Geschwindigkeit des Wandels wie auch in der sehr grossen Anzahl der vom Wandel erfassten (lautlichen, morphologisch-syntaktischen und lexikalischen) Sprach-Einzelmerkmale" äussere. Es hat sich gezeigt, dass besonders der Wortschatz dazu neigt, Konvergenzen mit grossräumigeren Varie- 
täten, seien es andere Dialekte oder die Standardsprache, einzugehen (cf. z. B. Wolfensberger 1967: 143; Christen 1998b: 60, 63). Haas (2000: 89) bezeichnet ihn daher als "Haupttummelplatz des Modernismus".

Im Vergleich der auf der Grundlage der SDS- und aus Online-Daten erstellten Karten konnten exemplarisch einige Entwicklungstendenzen erstmals weitgehend flächendeckend im schweizerdeutschen Sprachraum empirisch belegt werden:

Der auffälligste Trend in den Wortkarten zu Sommersprossen, Küchenzwiebel und Schmetterling bestand darin, dass sich ein Wort, das auch in der Standardsprache verwendet wird, zuungunsten der konkurrierenden Dialektwörter massiv ausgebreitet hat. Die überregionale Verwendung dieser Wörter in grossen Teilen des Sprachraums hat zu weniger klar strukturierten (Gesamt-) Kartenbildern geführt, als dies noch im SDS der Fall war. Der Vergleich der neuen Gesamt- und Einzelkarten hat gezeigt, dass diese standardsprachlichen Wörter zwar bereits in grossen Teilen des Sprachraums am häufigsten genannt wurden, die übrigen dialektalen Bezeichnungen bisher aber nicht haben verdrängen können (das Gebiet ihrer Nennung stimmt zum Teil sogar mit demjenigen des SDS überein, sie wurden nur weniger häufig genannt als die dominanten Wörter). Durch die Gegenüberstellung von Karten, die pro Ortspunkt eine Auswahl der Daten zeigen, und Karten, die alle Nennungen pro Ort zeigen, konnte aktueller Sprachwandel sichtbar gemacht werden. Je nachdem, wo die offensichtlich prestigeträchtigere Form zunächst verwendet wird, sind die Verbreitungsrichtungen unterschiedlich. Die Sommersprossen scheinen von Norden aus nach Süden vorgedrungen zu sein (wo sie etwas weniger häufig angegeben wurden), Merzefläcke haben sich in alle Himmelsrichtungen ausgebreitet, Zibele drängt von Westen nach Osten vor.

Mit der Ausdehnung von Merzefläcke lässt sich neben dem Trend zur Verwendung standardsprachlicher Wörter auch einer zur Übernahme dialektaler Wörter aus einem einflussreichen Nachbardialekt nachweisen (Regionalisierungstendenz). Neuschöpfungen wie Schmätzgi lassen darüber hinaus auf einen kreativen Umgang mit Wortschatzmaterial schliessen. Die verschiedenen Bewegungen können in derselben Region nebeneinander vorkommen.

In der Literatur wurde bereits auf eine Reihe zentraler Faktoren hingewiesen, die den Kontakt der Dialektsprechenden mit anderen Dialekten bzw. der Standardsprache in den letzten Jahrzehnten deutlich erhöht und damit Sprachwandel initiiert haben (cf. z. B. Wolfensberger 1967: 214; Hofer 1997: 28; Haas 2000: 87). Gesteigerte Mobilität innerhalb der Schweiz, ein grösserer Anteil städtischer Bevölkerung (mit unterschiedlichem dialektalen Hintergrund), moderne Massenmedien (mit Dialektsendungen bzw. -seiten) und Kommunikationsmittel (SMS, E-Mails oder Blogs im Dialekt) führen zu verstärktem Kontakt mit anderen (nicht nur Schweizer) Dialekten. Ein grösserer Anteil der Stadtbevölkerung hat in den Städten auch mehr Kontakte zur deutschen Standardsprache und eventuell auch zu anderen deutschen Dialekten, ebenso wie moderne Massenmedien (standardsprachliche Schweizer wie ausländische Sendungen bzw. Seiten), Kommunikationsmittel (in der Standardsprache), häufigere Kontakte zu Nicht-Dialektsprechenden (im In- wie im Ausland) und ein insgesamt erhöhter Anteil der Schriftlichkeit. Wie gross der Einfluss der einzelnen Faktoren ist, lässt sich empirisch schwer bestimmen und ist individuell sehr unterschiedlich. Dieser vielfältig initiierte Sprachkontakt führte in vielen Gebieten zu einem Nebeneinander von altem Dialektwort und neuer dialektaler oder standardsprachlicher Bezeichnung, wie durch einen Vergleich der Einzelkarten zu den dialektalen Bezeichnungen von Sommersprosse, Küchenzwiebel und Schmetterling sehr gut dokumentiert wird. Wenn die neuen Formen einen Selektionsvorteil aufweisen (z. B. weiträumigere Verständlichkeit, höherer Status), drängen sie die alten zurück. Dieser Vorgang des Sprachwandels ist in den gezeigten Beispielen unterschiedlich weit fortgeschritten. Wie das folgende Kapitel zeigt, gibt es Gruppen innerhalb der Sprachgemeinschaft, die entweder häufiger mit neuen Formen in Kontakt kommen und/oder eher gewillt sind, diese in ihre Sprache zu übernehmen.

\subsection{Einfluss soziodemografischer Faktoren}

In Übereinstimmung mit den bisherigen Untersuchungen zum Einfluss des Alters auf den Dialektgebrauch konnte statistisch sehr deutlich die grössere Neigung jüngerer Probanden zu Abweichungen vom SDS und damit flexiblerem Sprachverhalten nachgewiesen werden. Eine 
Visualisierung der Daten zeigt ausserdem sehr schön die nahezu lineare Abnahme der Tendenz zu einem moderneren Wortgebrauch mit zunehmendem Alter.

Überraschender war das Ergebnis, dass nur für die Sommersprossen-Daten ein Einfluss der Ortsansässigkeit (Ortsansässige zeigten weniger Abweichungen vom SDS) nachweisbar war. Das weist darauf hin, dass der Effekt der Ortsansässigkeit überschätzt wurde bzw. anders definiert werden müsste. Es gibt drei Möglichkeiten, warum die Ergebnisse dieser Arbeit teilweise von denen Wolfensbergers (1967: 214) abweichen: Sicherlich kommen in der Gegenwart auch die Ortsansässigen über mehrere der in Kapitel 5.2 genannten Faktoren in Kontakt mit anderen Schweizer Dialekten und/oder der Standardsprache, so dass auch sie in ähnlicher Häufigkeit vom SDS abweichen könnten wie die Nicht-Ortsansässigen. Das würde darauf hinweisen, dass der Faktor Ortsansässigkeit vs. Mobilität heute eine geringere Rolle spielt als bspw. in den 1960er Jahren. Oder aber diejenigen Probanden, die als nicht-ortsansässig eingestuft wurden, weil sie entweder nicht mehr im Gebiet der Ortsmundart wohnen, über die sie Auskunft gegeben haben, oder dort weniger als 10 Jahre wohnen, können (noch oder schon) sehr zuverlässig über diesen Dialekt Auskunft geben. Das würde heissen, ihre Kompetenz wurde unterschätzt und es würden weniger Jahre für eine Einstufung als "ortsansässig" ausreichen. Drittens reicht möglicherweise auch eine Ortsansässigkeit von 10 Jahren noch nicht aus, um signifikante Unterschiede hinsichtlich der Tendenz zu einem moderneren Wortgebrauch nachweisen zu können. Wird "Ortsansässigkeit" enger gefasst, bspw. indem auch die Eltern den Ortsdialekt sprechen müssen, hat der Faktor einen deutlich grösseren Einfluss. Das zeigt der für alle drei Datensätze geltende Zusammenhang des Dialekts der Eltern mit der Abweichung der Probanden vom SDS. Diejenigen Probanden, die angaben, dass ihre Mutter oder ihr Vater denselben Dialekt spricht, wichen weniger häufig von den SDS-Daten ab und erwiesen sich damit dialektal als konservativer. Um einen Anhaltspunkt für die tatsächliche Bedeutung dieser Erklärungsmöglichkeiten zu erhalten, wurden dieselben Tests noch einmal mit einer anderen Definition von Ortsansässigkeit, nämlich mit mindestens 20 Jahren Wohnsitz am Ortspunkt, durchgeführt. Die VGLM ergaben ebenfalls nur für den Sommersprossen-Datensatz einen signifikanten Einfluss der Ortsansässigkeit $(\mathrm{p}=0.01)$, wobei diejenigen Probanden, die mindestens 20 Jahre am Ort wohnten, eine geringere Abweichung vom SDS zeigten. Für die Küchenzwiebel- und Schmetterling-Daten konnte kein Effekt dieses Faktors nachgewiesen werden $(p>0.05)$. Dieses Ergebnis weist darauf hin, dass eher davon auszugehen ist, dass eine Kombination aus der ersten und zweiten Möglichkeit für das unerwartete Ergebnis zur Wirksamkeit des Faktors Ortsansässigkeit verantwortlich ist, d. h. der häufigere Sprachkontakt Ortsansässiger und die hohe (d. h. frühe bzw. lang anhaltende) Sprachkompetenz hinsichtlich der Zuweisung von Lexemen zu Dialekten die Bedeutung des Faktors Ortsansässigkeit verringert haben.

Kein Einfluss liess sich für das Geschlecht der Probanden nachweisen.

\subsection{Angemessenheit der Methode}

Die mehrfach angesprochenen Übereinstimmungen zwischen den Karten aus der SDS- und der Online-Erhebung sprechen dafür, dass die aktuellen wortgeografischen Verteilungen prinzipiell korrekt wiedergegeben werden, und rechtfertigen damit die Methodenkombination von direkter und indirekter Befragung. Das bedeutet auch, dass den Probanden (ähnlich wie den SDS-Gewährspersonen) zumindest im Wortschatz eine hohe Kompetenz zur Beurteilung wortgeografischer Gebrauchsgewohnheiten zugestanden werden kann.

Im Wortschatzbereich erweist sich die Online-Befragung damit als sehr reliable und valide Möglichkeit zum Nachweis dialektalen Wandels. Dank der zahlreichen Probanden konnte trotz der ungerichteten Probandenauswahl eine zufriedenstellende Ortsnetzdichte realisiert werden, so dass mit Hilfe von GIS-Karten wortgeografischer Wandel im schweizerdeutschen Sprachraum sichtbar gemacht werden konnte. Der Einsatz von wortgeografischen Verteilungskarten mit und ohne Datenauswahl erlaubt es ausserdem, aktuelle Wandelprozesse im Schweizerdeutschen sichtbar zu machen. Die hier vorgestellten exemplarischen Ergebnisse konnten einige Veränderungstendenzen im Deutschschweizer Raum aufzeigen, weitere Befragungen dieser Art können das Bild zumindest im Wortschatzbereich verdichten. 


\section{Literatur}

Bigler, Niklaus Jakob (1979): Mundartwandel im mittleren Aargau: Eine Untersuchung zu den heutigen Sprachverhältnissen im Spannungsfeld zwischen Ost- und Westschweizerdeutsch. Bern etc. (= Deutsche Sprache und Literatur 264).

Christen, Helen (1998a): Dialekt im Alltag. Eine empirische Untersuchung zur lokalen Komponente heutiger schweizerdeutscher Varietäten. Tübingen. (= Reihe Germanistische Linguistik 201).

Christen, Helen (1998b): "Convergence and Divergence in the Swiss German Dialects". Folia Linguistica 32/1-2: 53-67.

Christen, Helen (1988): Sprachliche Variation in der deutschsprachigen Schweiz: dargestellt am Beispiel der l-Vokalisierung in der Gemeinde Knutwil und in der Stadt Luzern. Stuttgart. (= Zeitschrift für Dialektologie und Linguistik: Beihefte N. F. 58).

Dräger, Marcel/Juska-Bacher, Britta (2010): "Online-Datenerhebungen im Dienste der Phraseographie". In: Ptashnyk, Stefaniya/Hallsteinsdóttir, Erla/Bubenhofer, Noah (eds.): Computergestützte und korpusbasierte Methoden in der Phraseologie, Phraseografie und der Lexikografie. Baltmannsweiler: 162-175.

Eichhoff, Jürgen (1982): "Erhebung von Sprachdaten durch schriftliche Befragung". In: Besch, Werner et al. (eds.): Dialektologie. Ein Handbuch zur deutschen und allgemeinen Dialektforschung. Berlin/New York: 549-554.

Elspaß, Stephan/Möller, Robert (2006): "Internet-Exploration: $\mathrm{Zu}$ den Chancen, die eine Online-Erhebung regional gefärbter Alltagssprache bietet". Osnabrücker Beiträge zur Sprachtheorie 71: 141-156.

Haas, Walter (2000): "Die deutschsprachige Schweiz". In: Schläpfer, Robert/Bickel, Hans (eds.): Die viersprachige Schweiz. 2., neu bearb. Aufl. Aarau etc.: 57-108. (= Sprachlandschaft 25).

Hofer, Lorenz (1997): Sprachwandel im städtischen Dialektrepertoire. Eine variationslinguistische Untersuchung des Baseldeutschen. Tübingen/Basel.

Hotzenköcherle, Rudolf (1986): "Zur Raumstruktur des Schweizerdeutschen: Statik und Dynamik". In: Schläpfer, Robert/Trüb, Rudolf (eds.): Dialektstrukturen im Wandel. Aarau: 33-69.

Hotzenköcherle, Rudolf (1962): Einführung in den Sprachatlas der deutschen Schweiz. Band A: Zur Methodologie der Kleinraumatlanten. Bern.

Juska-Bacher, Britta (im Druck): "SDS-Exploratoren und Online-Befragung - Lässt sich im Methodenmix ein Wandel in der Schweizer Dialektlandschaft nachweisen?" In: Christen, Helen et al. (eds.): Dialektologie: Wege in die Zukunft. Stuttgart. (= Zeitschrift für Dialektologie und Linguistik: Beihefte).

Niebaum, Hermann/Macha, Jürgen (2006): Einführung in die Dialektologie des Deutschen. Tübingen.

Schifferle, Hans-Peter (1995): Dialektstrukturen in Grenzlandschaften. Untersuchungen zum Mundartwandel im nordöstlichen Aargau und im benachbarten südbadischen Raum Waldshut. Bern etc. (= Deutsche Sprache und Literatur 1538).

Siebenhaar, Beat (2008): "Sprachwandel und Sprachgeografie - der Einfluss der Stadt Bern auf die Region". In: Krefeld, Thomas (eds.): Sprachen und Sprechen im städtischen Raum. Frankfurt am Main: 173-195.

Siebenhaar, Beat (2003): "Sprachwandel von Sprachgemeinschaften und Individuen". In: Häcki Buhofer, Annelies (eds.): Spracherwerb und Lebensalter. Tübingen/Basel: 313-325.

Siebenhaar, Beat (2000): Sprachvariation, Sprachwandel und Einstellung. Der Dialekt der Stadt Aarau in der Labilitätszone zwischen Züricher und Berner Mundartraum. Stuttgart. (= Zeitschrift für Dialektologie und Linguistik: Beihefte 108).

Sprachatlas der deutschen Schweiz (SDS). Begründet von Heinrich Baumgartner und Rudolf Hotzenköcherle. In Zusammenarbeit mit Konrad Lobeck, Robert Schläpfer, Rudolf Trüb und unter Mitwirkung von Paul Zinsli, hrsg. von Rudolf Hotzenköcherle (1962-1997). Bände I-VIII. Bern.

Trüb, Rudolf (1982): "Der Sprachatlas der deutschen Schweiz als Beispiel einer sprachgeografischen Gesamtdarstellung". In: Besch, Werner et al. (Hrsg.): Dialektologie: Ein Handbuch zur deutschen und allgemeinen Dialektforschung. Berlin/New York: 151-168. 
Wolfensberger, Heinz (1967): Mundartwandel im 20. Jahrhundert. Dargestellt an Ausschnitten aus dem Sprachleben der Gemeinde Stäfa. Frauenfeld. (= Beiträge zur schweizerischen Mundartforschung 14). 


\section{Anhang 1}

Veränderungskarten (Vergleich SDS- und Online-Daten) zu den dialektalen Bezeichnungen für Sommersprossen.

Erläuterung zu den Legenden:

Wird ein Wort sowohl im SDS als auch in der Online-Befragung genannt, werden die betreffenden Postleitzahlbezirke als "unverändert" bezeichnet.

Wird ein Wort im SDS angegeben, nicht aber in der Online-Befragung, sind die betreffenden Postleitzahlbezirke als "alt" markiert. ${ }^{20}$

Wird ein Wort im SDS nicht genannt, wohl aber in der Online-Befragung, sind die betreffenden Postleitzahlbezirke als "neu" gekennzeichnet.

\section{Sommersprossen}
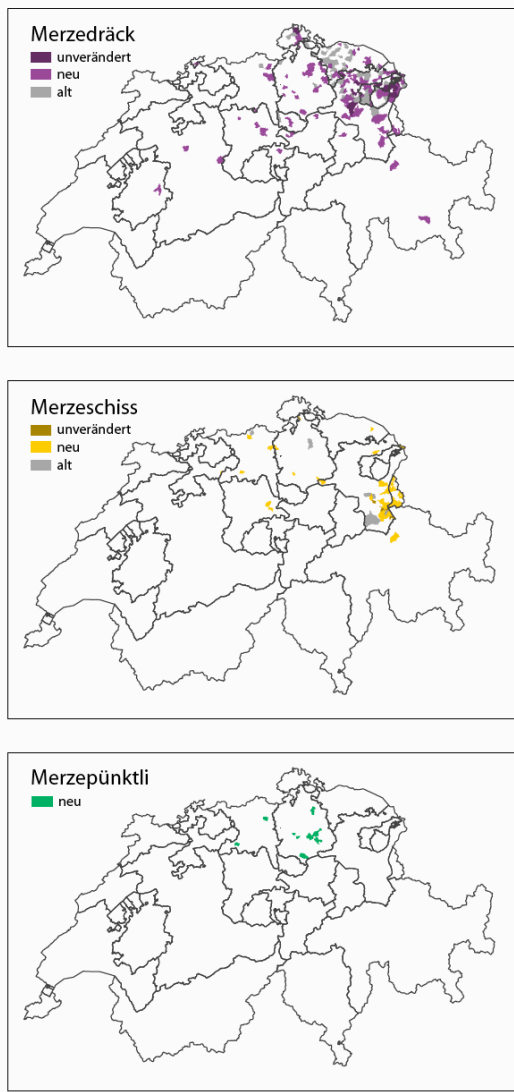
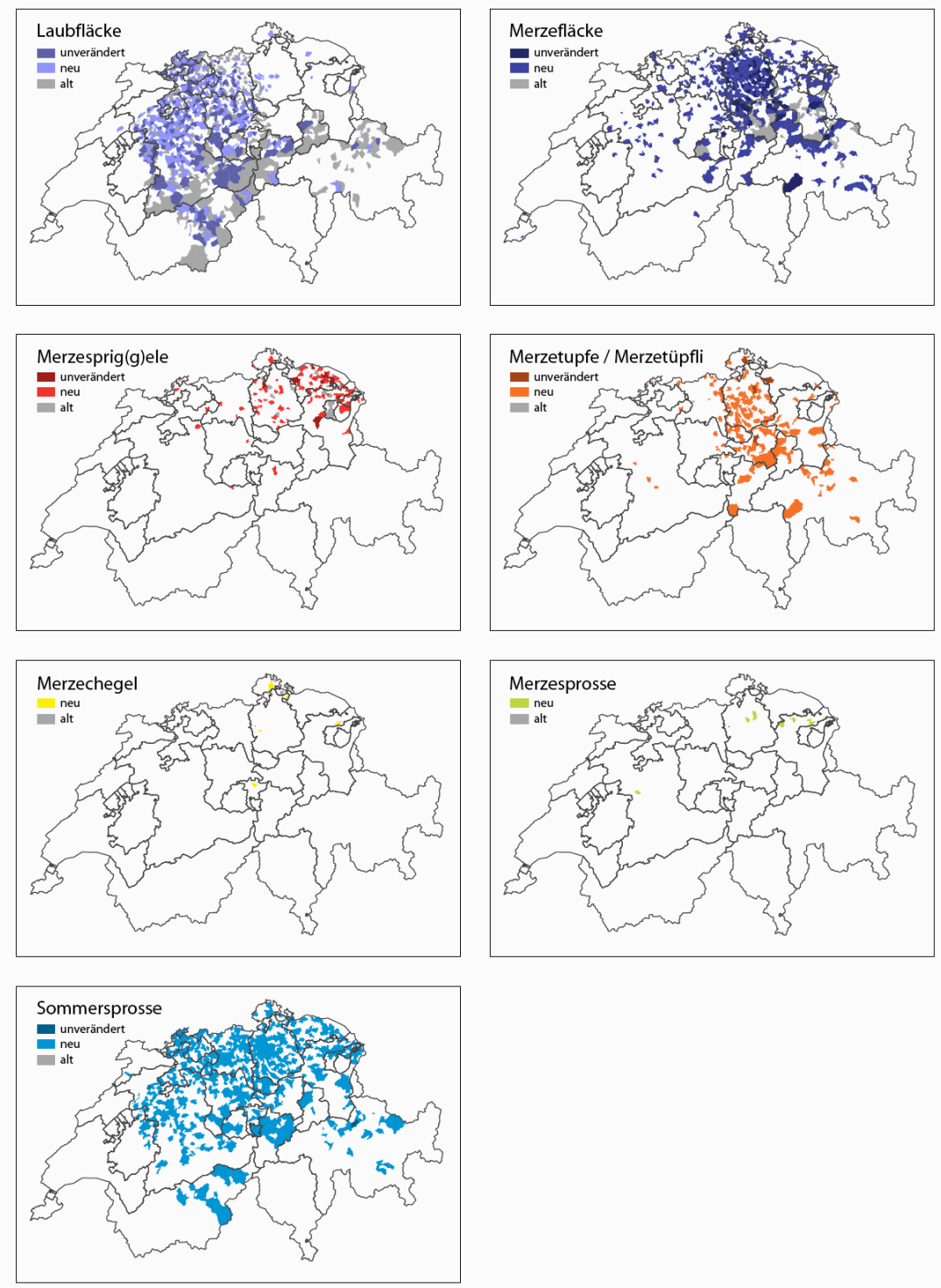

${ }^{20}$ Graue Flächen ("alt") können auch dadurch entstanden sein, dass Postleitzahlbezirke im SDS abgedeckt wurden, hingegen nicht in der Online-Befragung (der Deckungsgrad lag bei $85 \%$, cf. Kapitel 2). 


\section{Anhang 2}

Veränderungskarten (Vergleich SDS- und Online-Daten) zu den dialektalen Bezeichnungen für Küchenzwiebel.

Erläuterung zu den Legenden:

Wird ein Wort sowohl im SDS als auch in der Online-Befragung genannt, werden die betreffenden Postleitzahlbezirke als "unverändert" bezeichnet.

Wird ein Wort im SDS angegeben, nicht aber in der Online-Befragung, sind die betreffenden Postleitzahlbezirke als "alt" markiert. ${ }^{21}$

Wird ein Wort im SDS nicht genannt, wohl aber in der Online-Befragung, sind die betreffenden Postleitzahlbezirke als "neu" gekennzeichnet.
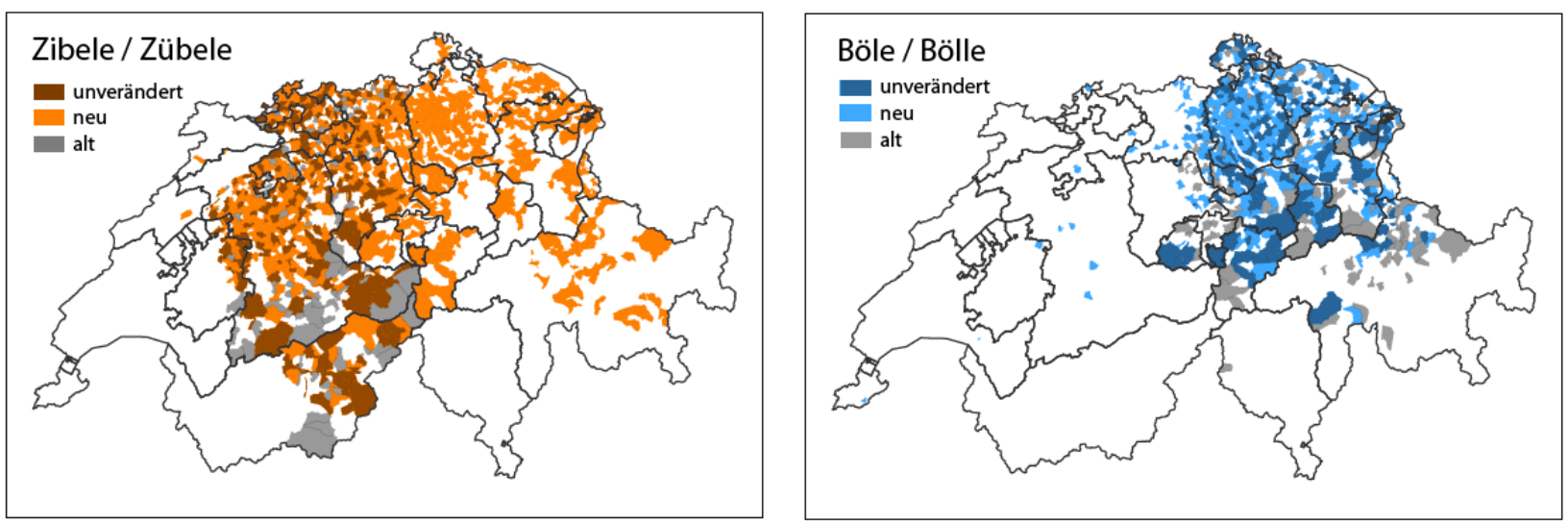

${ }^{21}$ Graue Flächen ("alt") können auch dadurch entstanden sein, dass Postleitzahlbezirke im SDS abgedeckt wurden, hingegen nicht in der Online-Befragung (der Deckungsgrad lag bei $85 \%$, cf. Kapitel 2). 


\section{Anhang 3}

Veränderungskarten (Vergleich SDS- und Online-Daten) zu den dialektalen Bezeichnungen für Schmetterling.

Erläuterung zu den Legenden:

Wird ein Wort sowohl im SDS als auch in der Online-Befragung genannt, werden die betreffenden Postleitzahlbezirke als "unverändert" bezeichnet.

Wird ein Wort im SDS angegeben, nicht aber in der Online-Befragung, sind die betreffenden Postleitzahlbezirke als "alt" markiert. ${ }^{22}$

Wird ein Wort im SDS nicht genannt, wohl aber in der Online-Befragung, sind die betreffenden Postleitzahlbezirke als "neu" gekennzeichnet.
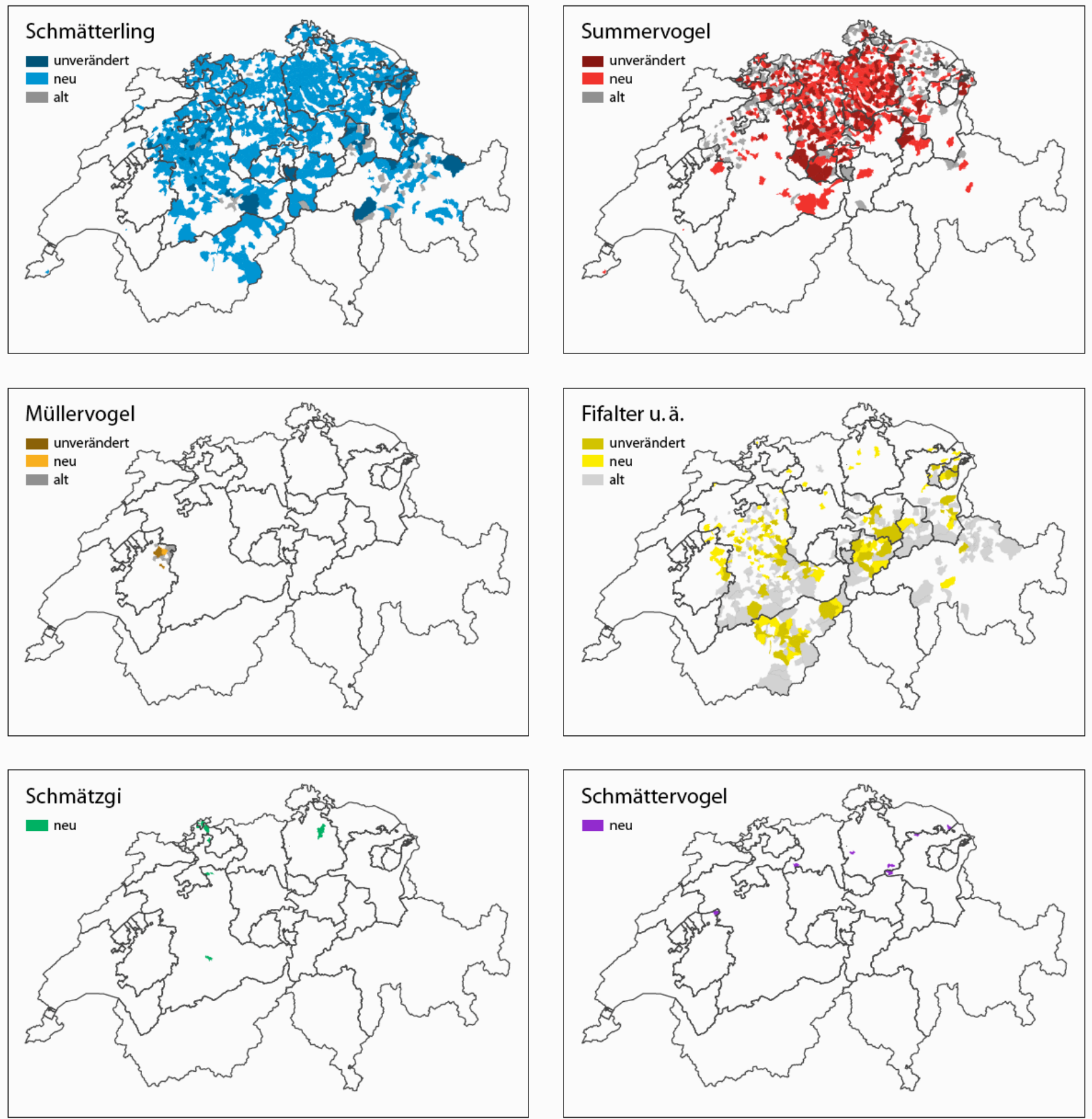

22 Graue Flächen ("alt") können auch dadurch entstanden sein, dass Postleitzahlbezirke im SDS abgedeckt wurden, hingegen nicht in der Online-Befragung (der Deckungsgrad lag bei $85 \%$, cf. Kapitel 2). 